BMC

Genomics

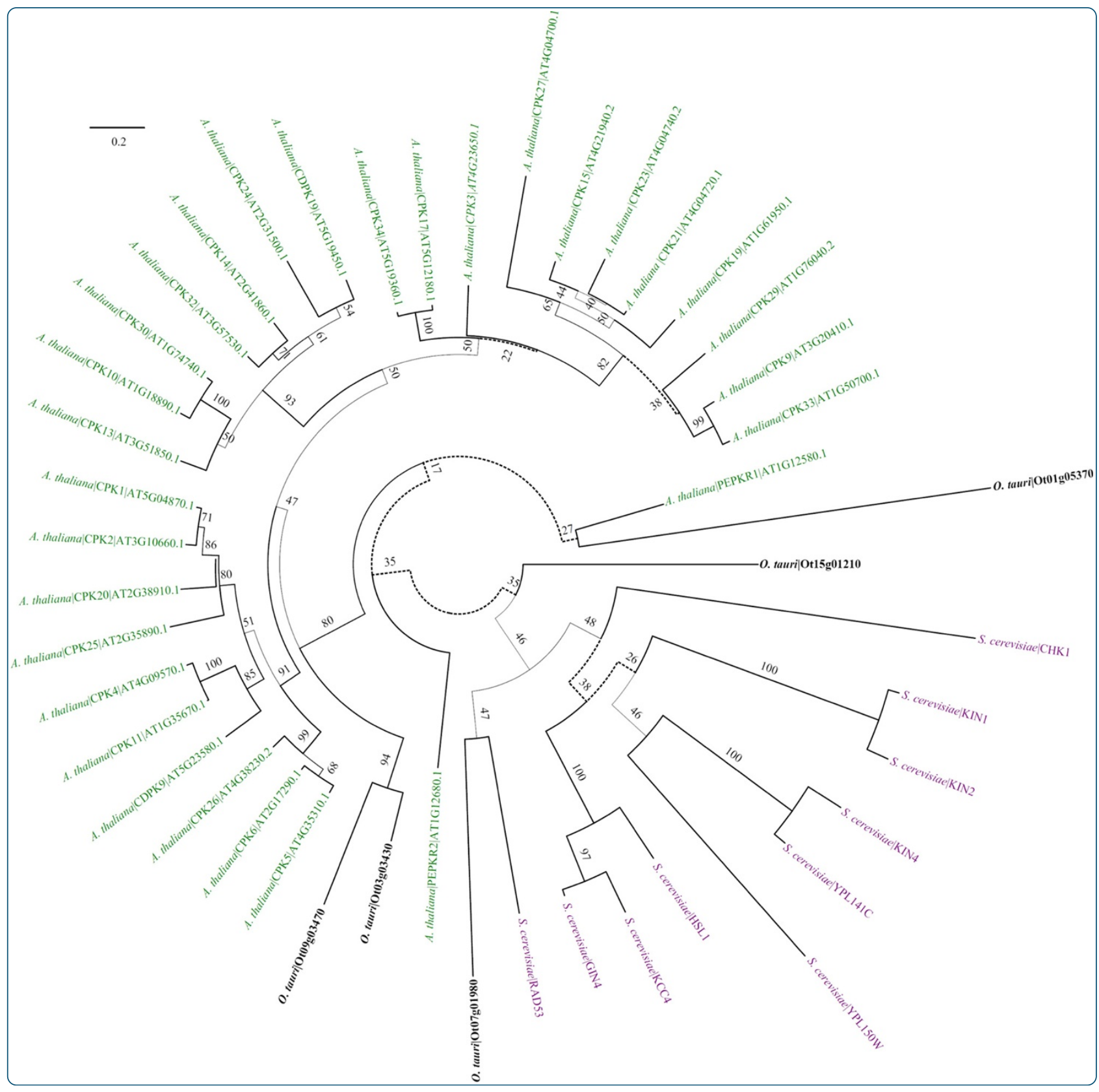

\title{
The reduced kinome of Ostreococcus tauri: core eukaryotic signalling components in a tractable model species
}

Hindle et al. 


\title{
The reduced kinome of Ostreococcus tauri: core eukaryotic signalling components in a tractable model species
}

\author{
Matthew M Hindle ${ }^{1,4}$, Sarah F Martin 1,2, Zeenat B Noordally,2, Gerben van Ooijen ${ }^{1,2}$, Martin E Barrios-Llerena ${ }^{1,2}$,
} T lan Simpson ${ }^{3,4}$, Thierry Le Bihan ${ }^{1,2}$ and Andrew J Millar ${ }^{1,2^{*}}$

\begin{abstract}
Background: The current knowledge of eukaryote signalling originates from phenotypically diverse organisms. There is a pressing need to identify conserved signalling components among eukaryotes, which will lead to the transfer of knowledge across kingdoms. Two useful properties of a eukaryote model for signalling are (1) reduced signalling complexity, and (2) conservation of signalling components. The alga Ostreococcus tauri is described as the smallest free-living eukaryote. With less than 8,000 genes, it represents a highly constrained genomic palette.

Results: Our survey revealed 133 protein kinases and 34 protein phosphatases ( $1.7 \%$ and $0.4 \%$ of the proteome). We conducted phosphoproteomic experiments and constructed domain structures and phylogenies for the catalytic protein-kinases. For each of the major kinases families we review the completeness and divergence of 0 . tauri representatives in comparison to the well-studied kinomes of the laboratory models Arabidopsis thaliana and Saccharomyces cerevisiae, and of Homo sapiens. Many kinase clades in $O$. tauri were reduced to a single member, in preference to the loss of family diversity, whereas TKL and ABC1 clades were expanded. We also identified kinases that have been lost in A. thaliana but retained in O. tauri. For three, contrasting eukaryotic pathways - TOR, MAPK, and the circadian clock - we established the subset of conserved components and demonstrate conserved sites of substrate phosphorylation and kinase motifs.
\end{abstract}

Conclusions: We conclude that $O$. tauri satisfies our two central requirements. Several of its kinases are more closely related to $\mathrm{H}$. sapiens orthologs than $\mathrm{S}$. cerevisiae is to $\mathrm{H}$. sapiens. The greatly reduced kinome of $\mathrm{O}$. tauri is therefore a suitable model for signalling in free-living eukaryotes.

Keywords: Conserved eukaryote signalling, Protein kinase phylogeny, Ostreococcus tauri, Model kinome, Phosphorylation, TOR signalling, MAPK cascade, Circadian clock

\section{Background}

Protein kinases are a major component of the complex signalling networks that coordinate all fundamental cellular processes, including transcription, cell cycle and metabolism. Protein kinases and phosphatases elicit reversible phosphorylation, which enable the rapid cellular responses that are crucial for survival in a continually changing environment. Protein kinases

\footnotetext{
* Correspondence: andrew.millar@ed.ac.uk

'SynthSys and School of Biological Sciences, University of Edinburgh, Edinburgh EH9 3JD, UK

${ }^{2}$ Institute of Structural and Molecular Biology, University of Edinburgh, Edinburgh EH9 3JR, UK

Full list of author information is available at the end of the article
}

activate and deactivate proteins by addition of the gamma-phosphate from ATP to serine $(\mathrm{S})$, threonine $(\mathrm{T})$, tyrosine $(\mathrm{Y})$, aspartate $(\mathrm{D})$ or histidine $(\mathrm{H})$ amino acid residues [1]. Cascades of consecutive kinase-mediated phosphorylation events constitute the backbone of signalling pathways [2]. The complexity of the signalling networks scales with size. Part of this complexity is constrained by the number of genes encoding protein kinases, also known as the kinome. The number of encoded protein kinases in free-living eukaryotes ranges from as little as 126 kinases in Saccharomyces cerevisiae [3] to 1000 in Arabidopsis thaliana [4]. Between these extremes, surveyed organisms include Dictyostelium discoideum

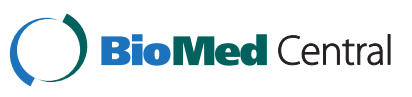

(c) 2014 Hindle et al.; licensee BioMed Central Ltd. This is an Open Access article distributed under the terms of the Creative Commons Attribution License (http://creativecommons.org/licenses/by/2.0), which permits unrestricted use, distribution, and reproduction in any medium, provided the original work is properly credited. The Creative Commons Public Domain Dedication waiver (http://creativecommons.org/publicdomain/zero/1.0/) applies to the data made available in this article, unless otherwise stated. 
with 285 kinases [5], the fruit fly Drosophila melanogaster with 251, and Homo sapiens with 518 kinases [6]. Minimal kinomes are present in parasites that are not obviously representative of other tractable species. The kinome of the parasitic fungus Encephalitozoon cuniculi [7] has only 32 kinases and lacks sequences that are ubiquitous in the kinomes of free-living eukaryotes, including the STE family, TOR and AMPK. E. cuniculi kinases are also highly divergent within fungi: 9 are reported to have no clear orthologs. The protozoan Giardia lamblia can be grown in pure culture and has a small genome of only 6,500 ORFs with a core of only 80 kinases, of which 14 have no clear orthologs and 5 are Giardia-specific [8]. The remainder of the kinome is composed of a large expansion of 198 Nek kinases, 139 of which are likely to be catalytically inactive. G. lamblia kinase domains were also found to have a mean sequence identity of only $40 \%$ with $H$. sapiens, lower than plant and fungal kinases (49-50\%).

It was originally thought that $\mathrm{S} / \mathrm{T}$ and $\mathrm{Y}$ kinases were unique to eukaryotes, and that bacteria and archaea operated a parallel system of $\mathrm{H}$ and $\mathrm{D}$ phosphorylation. However it is now known that S/T and Y phosphorylation is also important in both bacteria [9] and archaea [10]. While many eukaryote-like kinases (ELK) in bacteria share only remote sequence similarity with eukaryotic protein kinases (ePK) they share strong structural similarities $[11,12]$. The Rio and Bud32 families of kinases are common to both eukaryote and archaea [11]. Conversely, the Histidine kinases (HK) are also found in eukaryotes, where their roles include osmoregulation in several species [13] and ethylene hormone signalling in A. thaliana [14].

A well conserved 250 - 300 amino acid catalytic domain, known as the ePK domain [15], is present in most protein kinases and mediates protein phosphorylation. A small subset of kinases do not possess the ePK domain and are regarded as atypical protein kinases (aPK) [16]. As ePKs are structurally related, a common evolutionary ancestry, distinct from aPKs has been proposed [12]. Members of the protein kinase ePK family [1] are divided into the following major groups: AGC (named after protein kinases A, G and C), TK (Tyrosine Kinases), TKL (Tyrosine Kinase-Like kinases), CaMK (Calcium/ Calmodulin-dependent Kinases), CMGC (containing Cyclin-Dependent Kinases (CDK); Mitogen-Activated Protein Kinases (MAPK); Glycogen Synthase Kinase 3 (GSK3) and Cyclin-Dependent Kinase-Like (CKL)), CK1 (Casein Kinase 1), CK2 (Casein Kinase 2), STE (containing homologs of the yeast Sterile kinases), and AUR (Aurora Kinases). The TK family, particularly transmembrane receptor kinases, account for the majority of receptor kinases in humans and serve as cell-surface receptors for growth factors that trigger cell growth, proliferation and differentiation [6]. Non metazoan-eukaryotes, including the green lineage, do not possess genuine TKs [6]. Instead,
Y phosphorylation is substituted by dual-specificity kinases that phosphorylate S/T as well as Y $[17,18]$.

In this study we survey the kinase components of $O$. tauri and assess its suitability as a model organism for eukaryotic signalling, based on two criteria: (1) reduced signalling complexity and (2) conservation of signalling components. O. tauri is a promising candidate as it is the smallest free-living eukaryote [19], with a $12.6 \mathrm{Mb}$ genome, encoding 7,989 proteins with minimal genome duplication [20]. This reduced genome might impose simplified signalling. O. tauri is part of the Chlorophyta clade within the Plantae supergroup [21], and is taxonomically positioned at the base of the green-plant lineage. Given its size and taxonomic position, it is a promising candidate for generating hypotheses that can be transferred to more complex eukaryotes. O. tauri has a streamlined cell structure comprising a single nucleus, mitochondrion, Golgi body and chloroplast [22]. It possesses several benefits as an experimental model, cells can be readily and rapidly cultured in controlled laboratory conditions, where they undergo simple binary cell-division which can be synchronised by light/dark cycles. It has already been used as a model for the eukaryotic cell-cycle, helping to unify current understanding of cell-cycle regulation across eukaryotes [23]. The lack of a cellulose plant cell wall facilitates transformation [24,25] as well as organelle enrichment and protein extraction [26,27]. These genetic and proteomic tools have already been applied to studies of protein turnover [27], nutrient deprivation [26] and the plant circadian clock in experimental $[25,28,29]$ and mathematical approaches [30].

We survey the $O$. tauri kinome and examine conservation of protein sequences, through phylogenies of kinase orthologs in A. thaliana, $H$. sapiens and S. cerevisiae as the most widely studied models of plant, metazoan and fungal kinomes respectively. We then focus on three pathways, 1) TOR signalling in $H$. sapiens, 2) MAPKmediated GSK3 signalling in A. thaliana and 3) the core circadian clock. We evaluate the capacity of $O$. tauri components to support signalling in current models of these exemplar pathways. Building on our recent proteomic surveys $[26,31,32]$, we examine a large set of phosphorylated peptides detected by mass spectrometry and use these to validate phosphorylation-mediated signalling events in $O$. tauri. In combination with the phylogenetic evidence, we discuss the suitability of $O$. tauri as a model species to study protein kinase signalling.

\section{Results and discussion}

The overall proteomic similarity among O. tauri, A. thaliana, S. cerevisiae, and $H$. sapiens was revealed by a survey of shared ortholog-groups (Figure 1A) identified by OrthoMCL [33]. This approach allows a comparison of shared sequences, despite the widely differing number of 


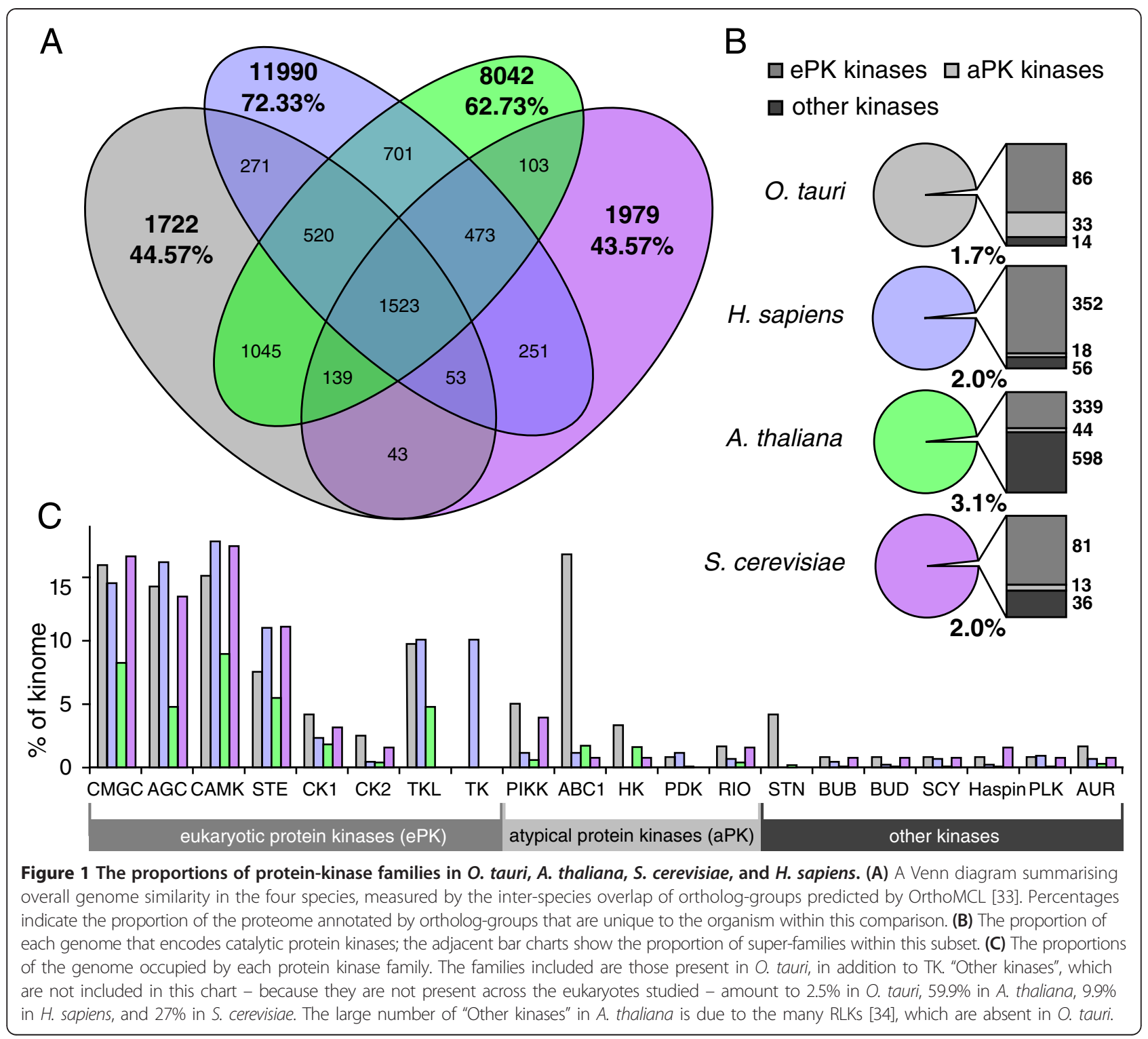

protein-family members in their proteomes. The proportion of ortholog-groups that were unique to O. tauri and S. cerevisiae was very similar, at $45 \%$ and $44 \%$ of their respective proteomes. O. tauri shares $10.6 \%$ of the 12,546 orthologgroups present in A. thaliana. The O. tauri genome contains a comparable number of $H$. sapiens protein families $(2,367)$ to the existing model species $S$. cerevisiae $(2,300)$.

We compared the number of protein kinases for each family in O. tauri with other model organisms, using an existing, high-level classification derived from 22 eukaryotic kinomes [35]. This data-mining approach was augmented by experimental identification of 5,563 phosphorylated O. tauri peptides from 107 liquid-chromatography-coupled mass spectrometry (LC-MS) experiments. These correspond to 3,994 uniquely identified phosphorylations of 2,214 peptide sequences of 1,252 proteins (Additional file 1:
Table S1), including several conserved protein kinases, discussed below. In the process of identifying and categorising kinases in $O$. tauri, we identified a novel gene locus, corrected 9 existing gene models, and patched sequencing gaps in 25 gene loci with sequence information from Ostreococcus lucimarinus data to generate a more complete database for peptide identification. Protein domain diagrams are attached as Additional file 2: Figure S1 while the new and patched gene models and sequences are detailed in Additional file 3: Figure S2. Evidence of phosphorylation motifs conserved between species is presented in Additional file 4: Figure S3.

\section{O. tauri protein kinase and phosphatase survey}

A survey of the 7,989 gene models [20] currently annotated in the $O$. tauri genome revealed 133 genes encoding 
catalytic protein-kinases and 32 protein phosphatases, respectively amounting to $1.7 \%$ and $0.4 \%$ of the known O. tauri loci (Figure 1B, Additional file 5: Table S2). The $O$. tauri kinome occupies a similar proportion of the genome to that found in S. cerevisiae (2\%, 130 kinases) [3] and $H$. sapiens (2\%, 426 kinases) [36] and is proportionally smaller than the A. thaliana kinome (3.1\%, 981 kinases) [37], with which it shares the greatest sequence similarity of components (Additional file 6: Figure S4).

Phosphatases, in contrast, do not scale with the size of the genome. The Human Phosphatase Portal (HuPho) [38] reports 135 protein phosphatases of which 107 are Protein Tyrosine Phosphatases (PTPs). The remaining 28 S/T phosphatases consist of two families, Metal Dependent Protein Phosphatases (PPMs or PP2Cs) and Phosphoserine Protein Phosphatases (PPPs). A. thaliana contains 131 phosphatases of which 10 are PTPs and the remaining S/T phosphatases contain 38 PPPs and 83 PPMs [37]. S. cerevisiae contains 25 protein phosphatases [3], which are composed of 6 PTPs, 12 PPPs and 7 PPMs. The O. tauri genome contains 32 protein phosphatases, which are composed of 8 PTPs, 10 PPPs and 14 PPMs. The higher proportions of S/T phosphatases to PTPs in $O$. tauri resemble the proportions found in higher A. thaliana more than $S$. cerevisiae and $H$. sapiens. The dominance of the PPM family within the S/T phosphatases in $O$. tauri is consistent with $A$. thaliana and $H$. sapiens but is in contrast to $S$. cerevisiae.

A categorisation of kinases into families by sequence similarity and phylogenetic analysis with the A. thaliana, $S$. cerevisiae, and $H$. sapiens kinomes confirmed the presence in $O$. tauri of all major ePK families (TKL, CaMK, CMGC, AGC, STE and CK1) present in the green lineage (Figure 1B). We also observed six small, conserved families of ePK-related protein kinases, which are classified as other-ePKs [6] and five families of aPKs. No Receptor-Like Kinases (RLKs) were found in O. tauri [39]. The main ePK families account for a large proportion of the kinome in all the eukaryotes. O. tauri contains 13 TKL-like kinases, which is consistent with a large expansion of this family in the green lineage [40]. In contrast the TKL family is absent in S. cerevisiae and many other fungal genomes [41]. For such a small kinome, $O$. tauri contains a surprising abundance of 20 ABC1-like kinases, which have few functionallycharacterised orthologs in other species [42-45]. Recent experimental technologies for targeted gene knock-out in $O$. tauri will therefore greatly assist in the elucidation of their function [46]. Within ePK subfamilies, not all branches are equally conserved, as is evident in the following phylogenetic analyses (Additional file 7: Figures S5, Additional file 8: Figure S6 and Additional file 5: Table S2).
The TOR pathway: PIKK, CMGC and AGC kinase families

Target of rapamycin (TOR) mediated signalling is vital to the regulation of growth and the key components exist throughout eukaryotes [47]. Here, we describe the phylogenetic relationships within the kinase families that participate in the TOR signalling pathway [48], aPK PI3K-related kinases (PIKK), and the ePK CMGC and AGC kinases.

\section{PI3K-related kinases (PIKK): TOR, ATR, ATM, TRRAP and DNA-PK}

A conserved family of cell-cycle control proteins, phosphatidyl-inositol-3-kinases (PI3Ks) are a class of kinases originally named after their ability to phosphorylate the 3'-hydroxyl group of phosphatidylinositols. The PI3Ks that also act as S/T protein kinases are called PI3Krelated kinases (PIKK). Six PIKKs are present in eukaryotic genomes. Several of these couple the DNA damage sensing and repair pathway with the control of cell-cycle checkpoints, thereby maintaining the genetic integrity of the genome [49].

The phylogeny of PIKKs (Figure 2) reveal that O. tauri contains four of the five PIKK family proteins that are present in plant genomes [50], namely TOR, Rad3related (ATR), Ataxia-Telangiectasia Mutated (ATM), transformation/transcription domain-associated (TRRAP) protein kinases. The fifth protein, SMG1, is absent in both O. tauri and A. thaliana, but is represented in Oryza sativa (rice). SMG1 has also been identified in 19 other members of the green lineage, and knockouts in Physcomitrella patens confirm a conserved role in the nonsense-mediated RNA decay pathway [51]. An additional ATR-like gene fragment (Ot02g03510) is also present in O. tauri but was omitted from the phylogeny analysis to prevent gaps in the alignment. The phylogeny consistently groups $O$. tauri sequences into the same clade as $A$. thaliana, distinct from the $S$. cerevisiae and $H$. sapiens group. The topology of the ATM, ATR, and TOR branches of the phylogenetic tree indicates that the $S$. cerevisiae proteins sequences have diverged considerably from $H$. sapiens. Unlike $A$. thaliana and $S$. cerevisiae, O. tauri additionally contains a DNAdependent protein kinase (DNA-PK, Ot12g01950), which groups with the $H$. sapiens DNA-PK in the PIKK phylogeny with an $88 \%$ bootstrap confidence (Figure 2). Within the green lineage, candidate DNA-PK orthologs outside the Chlorophyta could only be identified in P. patens (XP_001765725) and Selaginella moellendorffii (XP_002965996), suggesting that DNA-PK has been lost in higher plants. DNA-PK has a well-defined role in the Non-homologous DNA end-joining (NHEJ) pathway [52], and has recently been recognised in mammals as an important component in the stress-induced phosphorylation of Replication Protein A (RPA) [53]. RPA in turn forms a heterotrimeric complex, which interacts with 


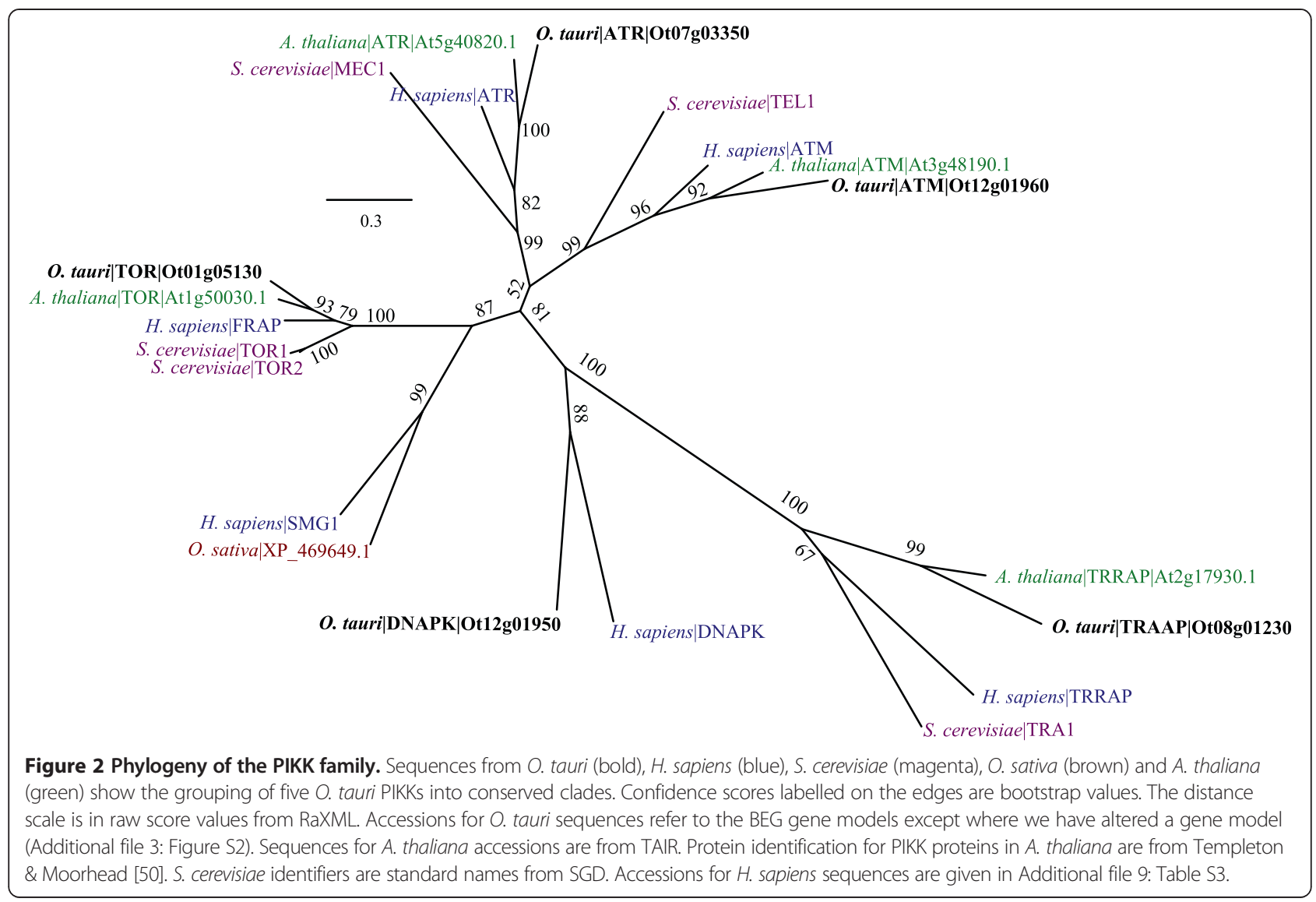

recombination components to repair DNA double-strand breaks. Unlike DNA-PK, RPA is conserved across eukaryotes, and phosphorylation sites on RPA have been found to be conserved in yeast, metazoa and higher-plants [54]. DNA-PK was also recently shown to be involved in innate immunity against viruses [55]. The presence of DNA-PK in the $O$. tauri kinome makes a first case for O. tauri as a model system to study protein kinases (balanced by absence of SMG1), in this case in DNA damage control and potentially in innate immunity.

\section{CMGC cell cycle family: CDK, MAPK, GSK3}

Among the most conserved of the ePKs are the cell cycle regulating CMGC kinases, which are named after their constituent subfamilies: CDK, MAPK, GSK3 and CLK. We identified $18 \mathrm{CMGC}$ kinases in O. tauri (Additional file 5: Table S2) and one CMGC-like gene. Seven are CDKs (Figure 3A) - two of these are CDK10 (Ot07g04140) and the closely related plant-like CDKG (Ot01g02660, Additional file 7: Figure S5A). The five further CDKs are the core cell cycle kinases CDKA (Ot04g00130), CDKB (Ot15g00680), CDKC (Ot01g04200) CDKD (Ot07g01260) and CDKE (Ot12g00510), which are present as single orthologs in O. tauri $[23,56]$, while up to 15 paralogs exist in A. thaliana, S. cerevisiae and H. sapiens (Figure 3A).
This makes $O$. tauri a powerful eukaryotic model to study a simplified cell cycle [23].

MAPKs are S/T-specific protein kinases, closely related to CDKs, their growth and stress-response functions including osmotic shock, oxidative stress and temperature response in plants have been extensively reviewed $[57,58]$. We identified 3 plant-like MAPKs in O. tauri (Ot08g00430, Ot09g04000 and Ot15g00120, Figure 3D, Additional file 7: Figure S5A), which have 8, 7 and 3 groups of paraologous genes respectively in A. thaliana. For MAPK (Ot08g00430) we observed phosphorylation of a conserved $\mathrm{Y}$ on the $\mathrm{T}-\mathrm{X}-\mathrm{Y}$ motif of the activation loop (Additional file 6: Figure S4A), which indicates conserved modes of activation. The greatly reduced set of MAPKs in O. tauri is a remarkable feature of a highly reduced kinome.

GSK3 is a highly conserved eukaryote CMGC kinase. The chaperone Heat Shock Protein 90 (HSP90) regulates the autophosphorylation of the activating $\mathrm{Y}$ in GSK3 [59]. Pharmacological evidence links both HSP90 and GSK3 with circadian timekeeping in O. tauri [28]. O. tauri, like other algae, has a single copy of GSK3 (Ot04g00510), compared to the ten found in A. thaliana [60] (Figure 3B). The O. tauri GSK3 kinase domain diverges considerably on its branch between $H$. sapiens and $A$. thaliana. 
Kindle et al. BMC Genomics 2014, 15:640

Page 6 of 20

http://www.biomedcentral.com/1471-2164/15/640

A

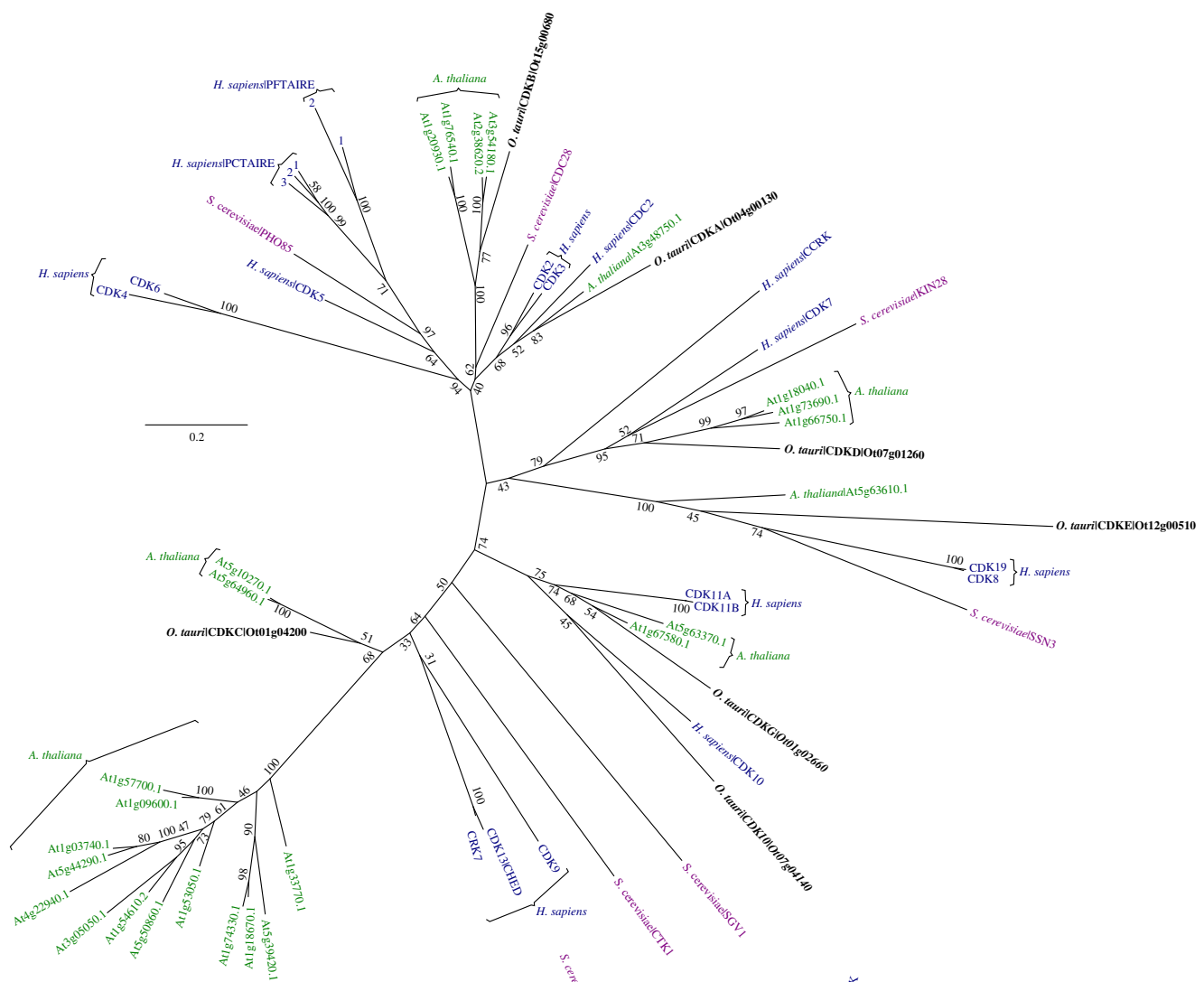

B

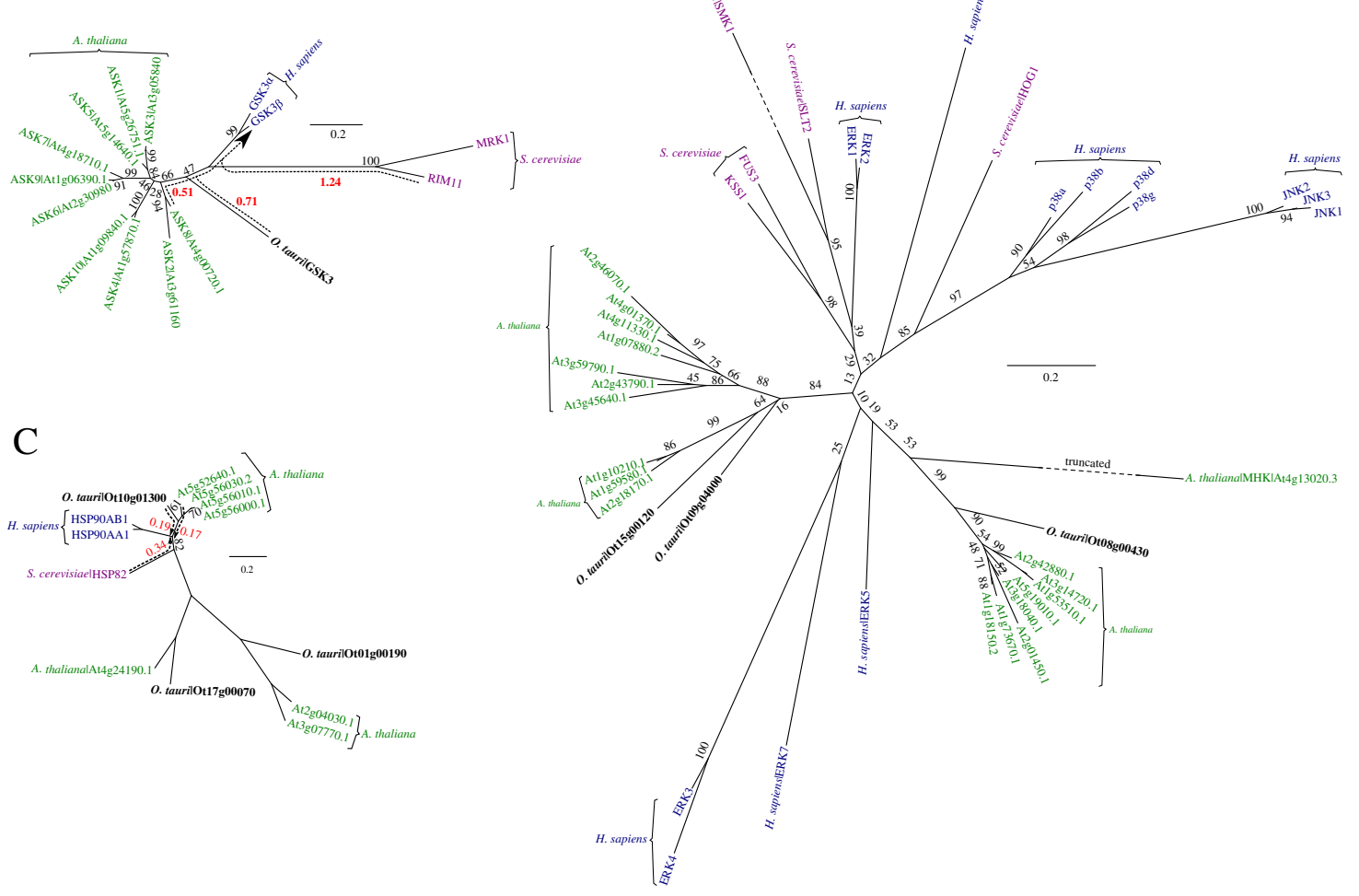

Figure 3 (See legend on next page.) 
(See figure on previous page.)

Figure 3 Phylogeny of CMGC kinases and HSP90. (A) Phylogeny of CDKs, showing the placement of previously identified cell-cycle kinases in O. tauri into the topology of a CMGC phylogeny. (B) Phylogeny of GSK3, highlighting the closer proximity of 0 . tauri and $A$. thaliana to the H. sapiens sequences (relative to S. cerevisiae). (C) Phylogeny of HSP90 chaperone, showing a similar topology to GSK3. (D) Phylogeny of MAPK, showing specialisation of $A$. thaliana MAPKs into two clades. A general explanation for phylogenies is in Figure 2. In (C) all omitted bootstrap values are 100\%. Dashed arrows (red) show the distance (sum of branch lengths) from the nearest A. thaliana, S. cerevisiae and O. tauri sequence to the point at which $H$. sapiens sequences diverge, in B-D.

However, the O. tauri GSK3 sequence is closer to H. sapiens (distance 0.71 ) than S. cerevisiae (1.24). O. tauri also contains a single ortholog candidate for HSP90 (Ot10g00440) (Figure 3C), while A. thaliana has four HSP90 paralogs [61]. Two closely HSP90-related clades in Figure 3C, acting as outgroups to confirm HSP90 orthology, reveals further $A$. thaliana specialisation of HSPs that is shared in the O. tauri genome.

\section{AGC Kinases: PDK1, S6K and PKG}

Members of the AGC family are cytoplasmic S/T kinases (named after PKA, PKG, and PKC), some of which contain $\mathrm{Ca}^{2+}$ sensing domains, regulate glycogen metabolism and ion channel conductance. Phylogeny across diverse eukaryotes has revealed a complex patchwork of conservation that suggests a history of successive contractions and expansions in the AGC kinases [62]. One of the few constants across eukaryote lineages is the PhosphoinositideDependent Kinase-1 (PDK1). Within A. thaliana only PDK1, Nuclear Dbf2-Related (NDR), Ribosomal S6 Kinase (S6K or RSK) are conserved [63]. Similarly, within O. tauri PDK1 (Ot03g02170, Figure 4A), S6K (Ot07g02590, Figure 4D), and NDR (Ot09g00870) are also conserved. Existing work has revealed an expansion of S6K and NDR into large and ubiquitous families across the kinomes of higher plants [40]. PDK1 is present throughout the green lineage but many orthologs, including $O$. tauri, lack a functional lipid-binding domain [62]. PKA- and PKG-like kinases exist in higher plants and other Streptophytes [40] but these have diverged substantially: orthologs from the Chlorophyta such as O. tauri have closer sequence similarity to metazoan and fungal sequences than the closest sequences in Streptophytes have to any of these groups. The most PKA- and PKG-like kinase sequence in O. tauri is Ot02g05760 (Figure 4B and C). The plant specific light sensor Phototropin1 (PHOT1, Ot16g02900) and Incomplete Root hair Elongation (IRE) (Ot09g04120) kinases are also present. O. tauri contains 13 AGC and four AGC-like kinases, of which 5 contain cNMP-binding domains (Additional file 7: Figure S5B).

PDK1 is the most important member of the AGC family in terms of phylogeny as it represents a highly conserved kinase, which has changed little since the divergence of eukaryotic AGCs [62]. PDK1 in O. tauri is most similar to the two A. thaliana orthologs (1.26). It also groups closer to $H$. sapiens (1.67) than $S$. cerevisiae orthologs (1.81) (Figure 4A). PDK1 is thought to be a basal conserved kinase, which predates the divergence of ePKs [62], and is therefore used to root AGC phylogenies (Figure $4 \mathrm{C}$ and D). PDK1 has also been termed the 'master kinase' of AGC signal transduction [64] because of its critical role in cellular survival through the activation of Protein Kinase B (PKB, also known as Akt) and S6K in humans [65]. However, out of these two PDK1 targets only S6K (Figure 4D) is conserved in A. thaliana and $O$. tauri.

The cAMP-dependent protein kinases (PKAs) and cGMP-dependent protein kinases (PKGs) are part of the same sub-family of kinase domains [36] and have similar domain components, and quaternary structure [66]. PKG is composed of a single protein with cGMP binding and protein-kinase activity. PKA is a heterodimer composed of separate protein-kinase and cAMP binding subunits. The inactive complex disassociates when cAMP binds to the regulatory subunit, which releases the active protein-kinase component [67]. As with many AGC proteins, a conserved C-terminal tail acts as a phosphorylation site for priming the protein-kinase active-site [67]. There are five AGC kinases with cNMP binding domains in O. tauri. Two of these (Ot02g05760 and Ot13g01150) contain all three domain components and have kinase domains with the strongest similarity to $H$. sapiens PKAs (Figure $4 \mathrm{~B}$ and Additional file 2: Figure S1). Ot13g01150, has the closest domain structure to $H$. sapiens PKA/PKG, and appears at the base of a subclade with two other kinases (90\% confidence), branching prior to the divergence of PKA and PKG (Figure 4C).

Ot02g05760 is assigned with low confidence (59\%) to the base of the PKG branch. However, it diverges near to the root of $H$. sapiens PKA-like kinases, which results in the domain being closer to PRKX (1.28) than to PKG (1.30); PRKX is part of the family of PKA catalytic subunits [68]. This supports a PKA like activity for the domain, rather than the more constrained PKG substrate specificity [69].

\section{The minimal TOR Pathway in O. tauri: An inventory}

TOR is highly conserved across eukaryotes and acts as a master regulator for nutrient-responsive growth in yeast, metazoa [70], and plants [71]. S6K1 and S6K2 are targets of the TOR pathway in A. thaliana [71], and rapamycin 
A

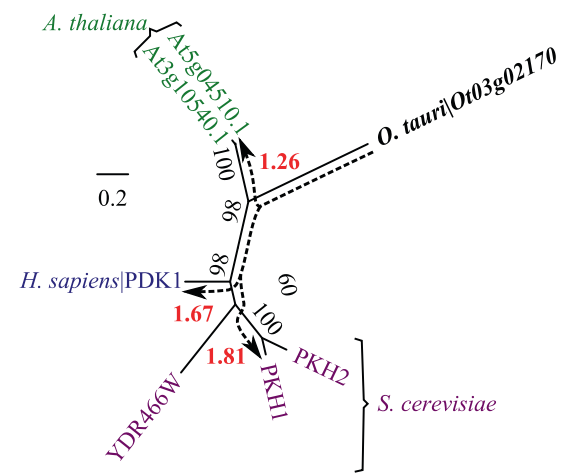

B

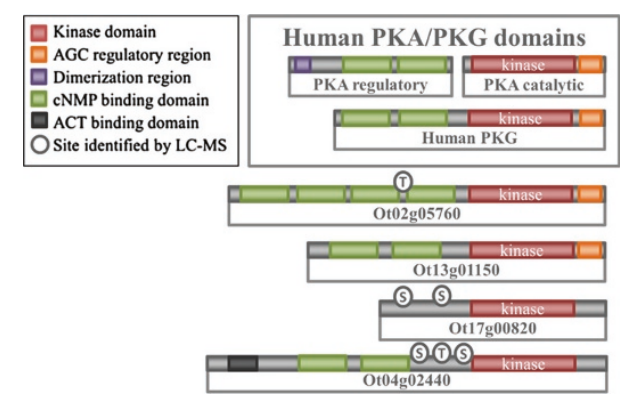

C

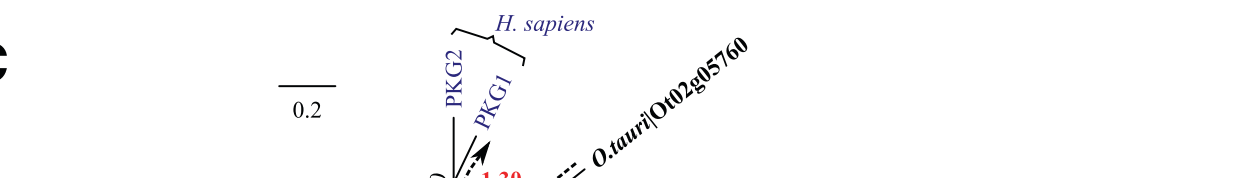

D

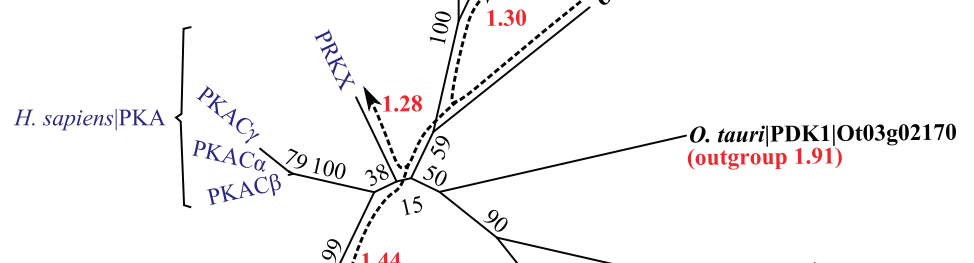

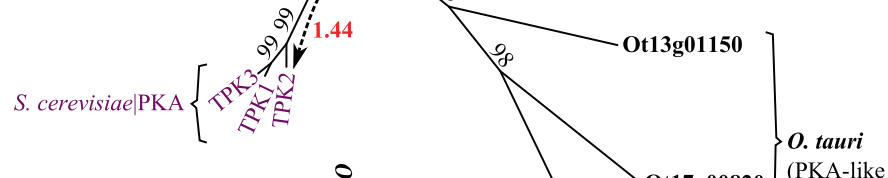

D
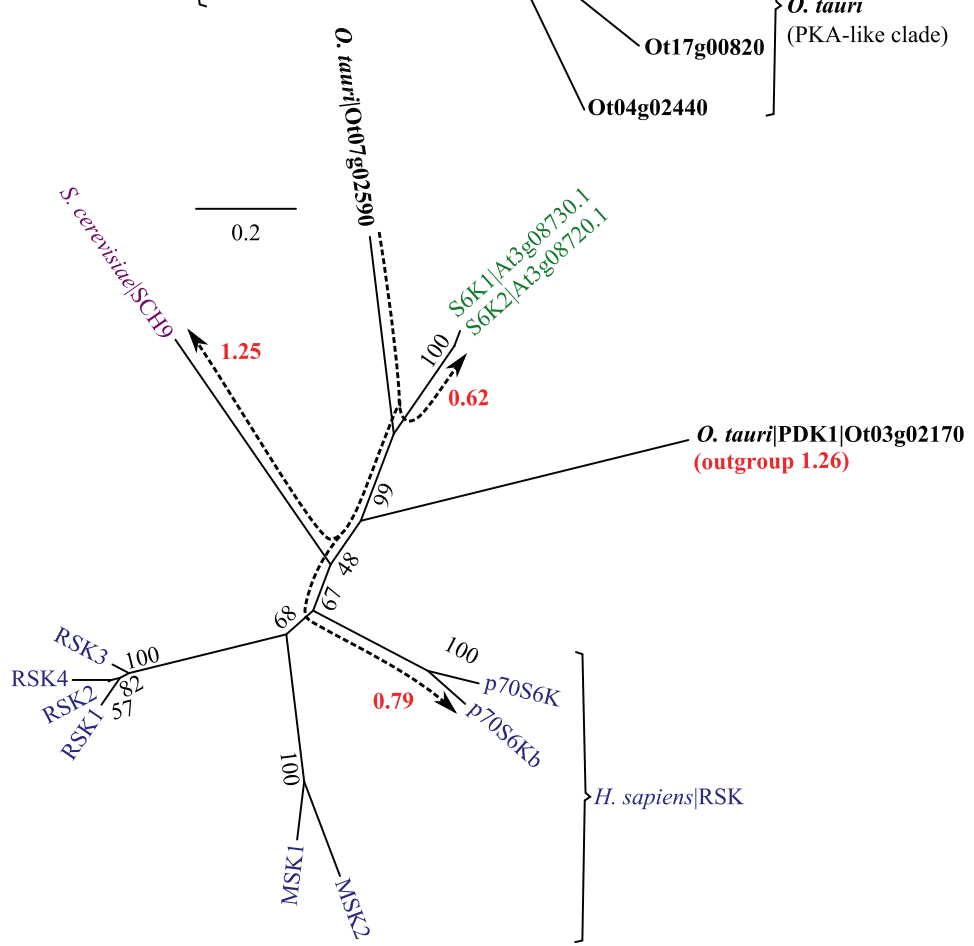

Figure 4 (See legend on next page.) 
(See figure on previous page.)

Figure 4 Phylogeny of AGC kinases. (A) Phylogeny of the PDK1 master regulator. (B) Comparison of domain arrangement for PKA- and PKG-like kinase in H. sapiens and O. tauri. (C) Phylogeny of kinase domains for PKA- and PKG-like proteins and (D) S6K phylogeny as compared to the S. cerevisiae ortholog (SCH9) and the H. sapiens RSKs. A general explanation for phylogenies is in Figure 2. Dashed arrows (red) show distances (sum of branch lengths). The distances shown are from $O$. tauri kinases to their nearest orthologous sequence in each species. S. cerevisiae identifiers are standard names from SGD.

inhibits this pathway, as in other organisms. S6K contains a conserved C-terminal motif that is a target for TOR phosphorylation and PDK1 binding, and this motif is highly conserved in O. tauri. In mammals, complexes of mTOR with RAPTOR (TORC1) and RICTOR (TORC2) mediate distinct signalling pathways. The LST8 protein is a common component of both complexes. Equivalents for both mTOR complexes exist in yeast $[72,73]$. O tauri, like the rest of the green lineage, only contains components of TORC1. The kinase targets of TORC2 (PKB, PKC) are absent from O. tauri, and across the green lineage. In contrast, $\mathrm{S} 6 \mathrm{~K}$ is a conserved as a target of TORC1 in the green lineage [71], suggesting that the TORC1-containing mTOR complex could be the prototypical pathway for TOR signalling.

Having established the phylogenies for the essential TOR pathway components GSK3, TOR, PDK1 and S6K [48,74-76] and its essential regulators in O. tauri, we now turn to analysing the complex series of phosphorylation events of the TOR pathway. The conserved O. tauri components required for GSK3-mediated S6K regulation are shown in Figure 5, overlaid with the mammalian interactions based on conserved phosphorylation motifs and binding sites. It has been suggested that GSK3 may initiate the activation process of S6K [48]. This involves a complex series of phosphorylation events by multiple components. Activated S6K transmits the final TOR pathway signal by phosphorylating the ribosomal protein S6, initiating it to regulate the translation machinery. Upstream, the activation of S6K is proposed to require the concerted action of three phosphorylation events by GSK3, the TORC1 complex and PDK1, in this order.

In mammals, the first of these phosphorylation events is by GSK3 to the equivalent residue of S398 on the S6K turn motif [48]. The GSK3 target S/T-X-X-X-S/T motif at this site is conserved in $S$. cerevisiae, A. thaliana, and O. tauri (Additional file 4: Figure S3B). This residue is constitutively phosphorylated in mammals, and is a dephosphorylation target of PP2C. The presence of GSK3 is proposed to infer resistance to $\mathrm{PP} 2 \mathrm{C}$-mediated inactivation of S6K by countering dephosphorylation [48]. The phosphorylation of S6K by GSK3 at S398 is a pre-requisite for the subsequent phosphorylation of the C-terminal T415 by TOR. The phosphorylation by TOR in turn enables the binding of active PDK1 [77] to the C-terminal motif. The activation of human PDK1 requires autophosphorylation of a $S$ in the activation loop, which is also a 14-3-3 binding motif [78]. Human 14-3-3 binds to the phosphorylated motif in PDK1 [79]; 14-3-3 also regulates PDK1 in A. thaliana [80]. The activation-loop $\mathrm{S}$ is conserved in $A$. thaliana and O. tauri PDK1 (S210). There are only two 14-3-3 proteins in O. tauri (Ot18g01040 and Ot08g00720), providing a limited number of candidates for PDK1 regulation. Active PDK1 binds to the primed TOR motif at the C-terminus of S6K (T415). This allows PDK1 to phosphorylate a T residue in the S6K activation loop [74,75], which is also conserved in $A$. thaliana and $O$. tauri (S260). Yeast and human TOR phosphorylates TAP42 (or $\alpha 4$ in humans), which affects the formation of a TAP42:PP2A complex [81]. PP2A has been shown to dephosphorylate S6K [82], but it is unclear what role this potential signalling pathway has on $\mathrm{S} 6 \mathrm{~K}$ regulation in higher eukaryotes [83]. Both TAP42 (Additional file 4: Figure S3) and PP2A (Ot07g01700) are found within O. tauri and the green lineage, though the TAP42 ortholog in O. tauri was previously unannotated.

We have shown conservation of the AGC kinases in the TORC1 pathway in O. tauri. Key phosphorylation motifs and binding sites are also conserved, for all the components of the model proposed by Shin et al. [48]. No phosphorylation was detected for the three key residues of S6K in our phosphoproteomic surveys. S6K was present, as phosphorylation at S61, S65, and S76 were detected and similar samples observed the unphosphorylated protein [26]. However, the lack of detected phosphorylation in $O$. tauri cannot be taken as contrary evidence as the quantity of observed phosphorylation in proportion to expected phosphorylation in O. tauri is still relatively low. For example, 28 phosphorylations of human S6K are currently known [69], and assuming a similar quantity of modification in $O$. tauri we have observed in the order of $10 \%$ of phosphorylations.

\section{The CaMK family: CPK and SnRK1 (ePK)}

The CaMK are an important family of S/T-specific kinases with functionally divergent roles, which are represented by five O. tauri genes and thirteen CaMK-like genes that are more distantly related (Additional file 5: Table S2). Consistent with previous findings [84], plant CaMKs are highly divergent with yeast and human proteins. Hence, we sub-classified the O. tauri CaMKs based 


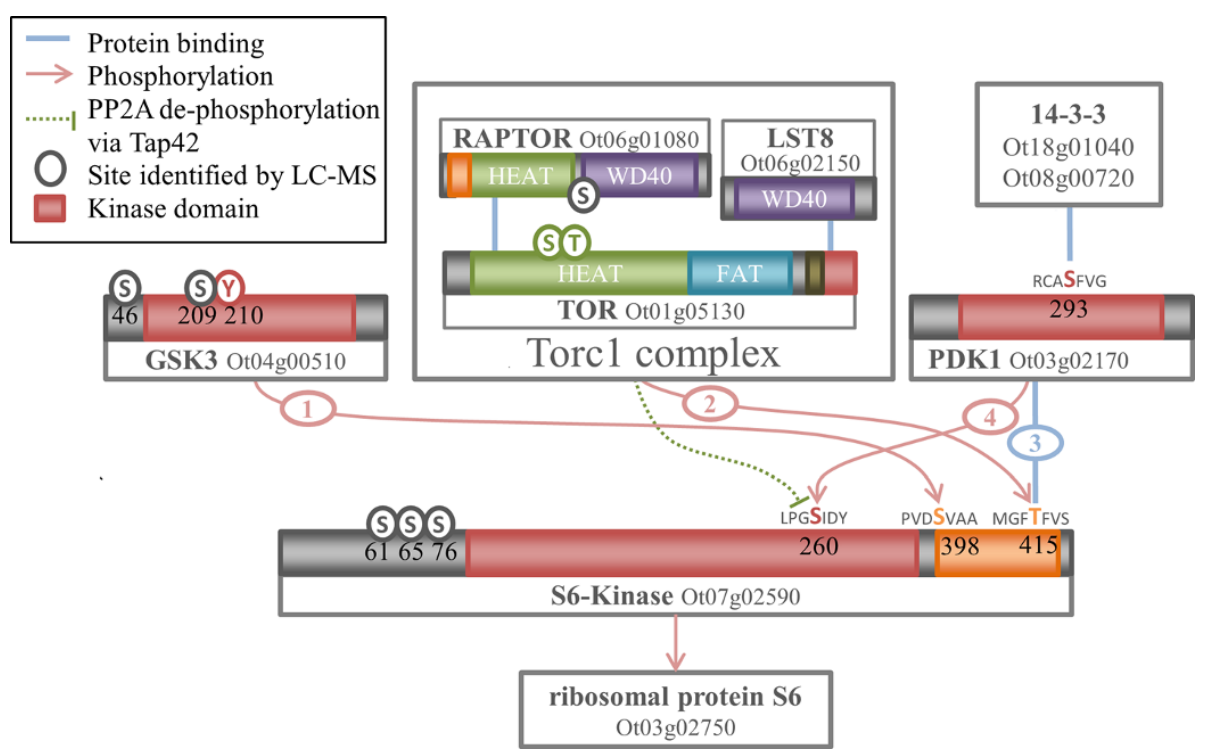

Figure $\mathbf{5}$ Schematic of the regulation of TOR signalling and S6K activation in $\mathbf{O}$. tauri. Numbered connectors indicate the order of the stages of S6K activation, as proposed by Shin et al. [48]. (1) GSK3 phosphorylates S398 allowing the (2) phosphorylation of T415 by the TORC1 complex. (3) Phosphorylated T415 is able to bind PDK1, which phosphorylates S260, leading to the activation of S6K. Additionally, TOR mediated inhibition of S6K is shown, which may occur via TAP42, previously unannotated in the 0 . tauri genome. TAP42 in yeast binds PP2A and prevents the latter from dephosphorylating the GSK3 target site of S6K.

on the separate phylogenies of two plant sub-families: Calcium-Dependent Protein Kinases (CDPK; Additional file 7: Figure S5C) and Sucrose-nonfermentation1-Related protein Kinases (SnRK, Figure 6A; Additional file 7: Figure S5D). In plants CaMKs have a large diversity of roles in extracellular signalling and target substrates in pathways such as carbon and nitrogen metabolism, homeostasis, transcription and proteasome regulation [85].

SnRK are an important subfamily of conserved CaMKs, which are related to SNF1 in yeast. SnRK1 kinases are the founding members and are most closely related to SNF1. It is also the only member of the SnRK family which is present across all eukaryotes and core members are involved in energy regulation in the cell $[86,87]$, with a primary function in glycogen metabolism [88]. The O. tauri SnRK1 (Ot06g03970) is most closely related to the A. thaliana SnRK1s (Figure 6A; Additional file 7: Figure S5D). In addition to the core SnRKs, A. thaliana contains a functionally diversified set of SnRK subfamilies [86], which are absent from O. tauri. The human ortholog 5' AMP-activated Protein Kinase (AMPKa) is equidistant to O. tauri and the S. cerevisiae ortholog SNF1, indicating O. tauri may also be a suitable model for SnRK signalling in humans (Figure 6A).

CDPKs have many roles in biotic and abiotic signalling pathways [85]. Two kinases exist in O. tauri (Ot09g03470 and Ot03g03430) with clear CDPK domain architectures and kinase domains. These have recently been classified as group I algal CDPKs [89]. CDPKs are typically a large family, involved in a variety of roles specific to higher plants, including herbivore defence [90] and abscisic acid signalling [91-93]. These specialised functions indicate that kingdom-specific adaptations have driven the sequence diversity of CDPKs. A Phosphoenolpyruvate carboxylaseRelated Kinases (PEPKR) Ot01g05370 is also present in $O$. tauri (Additional file 7: Figure S5C). Two other O. tauri kinases align more closely to the $S$. cerevisiae RAD53 (Ot15g01210 and Ot07g01980) than CDPKs. Ot15g01210 has previously been classified as a group IV algal CDPK [89]. Recent CDPK phylogenies by Hamel et al. [89] have shown that plant CaMKs are likely to have diverged between the emergence of group IV and I, which places Ot15g01210 and Ot07g01980 within two distinct clades for plant CDPKs. The retention of group IV and I clades within the reduced $O$. tauri kinome suggests an important conserved role for these kinase in calcium signalling.

\section{The MAP2K pathway and STE kinase family}

The downstream effects of extracellular signals, which are mediated by kinases such as the CaMKs and AGCs, are the MAPK cascades. These form signalling connections from the cellular environment into the nucleus, in order to affect transcriptional changes [2]. All but the final target of the MAPK cascade are found within the STE family. Here we examine the STE family of kinases with a view to understanding an exemplar MAPK pathway from $A$. thaliana: the GSK3-mediated regulation of stomatal opening through a MAP2K target $[94,95]$. As for the previously described TOR pathway, we first assess the relevant kinase orthologs in O. tauri. 


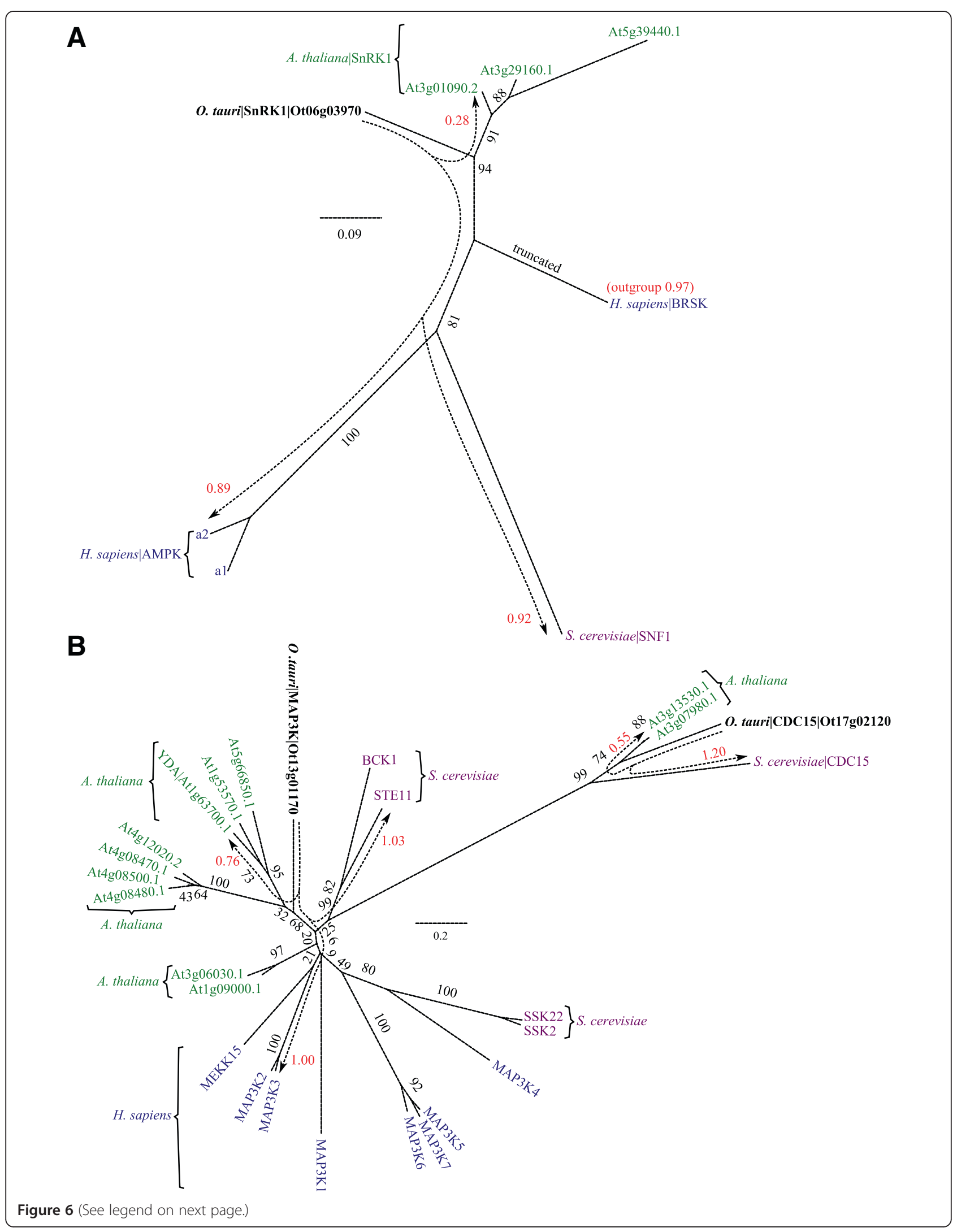


(See figure on previous page.)

Figure 6 Phylogeny of SnRK1s and MAP2Ks. (A) Phylogeny of SnRK1 showing orthology with SNF1 in S. cerevisiae and AMPKs in H. sapiens.

Brain-Specific Kinase (BRSK) acts as an outgroup. The full set of SnRKs are reported in Additional file 7: Figure S5D. (B) Phylogeny of MAP3K revealing distinct YDA- and CDC15-like MAP3Ks in O. tauri. It shows a strong conservation within the S. cerevisiae and plant cell cycle kinase CDC15 (not present in H. sapiens or other metazoa). The large numbers of MAP3K proteins in other species are reduced to a single member in 0 . tauri. A general explanation for phylogenies is in Figure 2. Raw branch lengths (red) are annotated to broken-line arrows and show the distances from 0 . tauri kinases to their orthologous sequences in A. thaliana, S. cerevisiae, and H. sapiens. S. cerevisiae identifiers are standard names from SGD.

\section{STE kinases}

The STE kinases contain the MAP4K, MAP3K and MAP2K components of the MAPK cascade [6]. Eight STE family kinases and an additional six STE-like kinases were identified in O. tauri (Additional file 7: Figure S5E). These include one MAP2K (Ot04g04050), two MAP3K (Ot13g01170 and Ot17g02120, Figure 6B), and two MAP4K (Ot02g05830 and Ot13g02030) kinase candidates. In contrast, $A$. thaliana has 10,11 and 7 orthologous genes respectively, again emphasizing the potential of $O$. tauri as an experimental model for gene manipulation in MAPK signalling studies. Within MAP3Ks Ot13g01170 is the only member of the MEKK clade and Ot17g02120 is a CDC15-like protein. The STEs are closely related to the Tyrosine-Kinase like (TKL) family, and contain the plant-RAF kinase, which also act as MAP3Ks [96]. Ot12g01310 is the only confirmed plantRAF kinase (Additional file 5: Table S2). It contains a Constitutive Triple Response 1 (CTR1) domain (Additional file 2: Figure S1), confirming it as an ortholog of the CTR1 gene: a potential-MAP3K that in A. thaliana is negatively regulated by the ethylene responsive histidine kinase ETR1 [97].

O. tauri also contains a single plant-like APG1 kinase (Ot06g01800) with four orthologous proteins in A. thaliana. C-terminal phosphorylation of APG1 was observed in O. tauri. APG1 kinases in yeast and A. thaliana are a target for the negative regulation of autophagy by TOR $[98,99]$, highlighting another conserved facet of the TOR pathway.

\section{The minimal MAP2K Pathway in O. tauri}

The brassinosteroid signalling pathway acts upstream of GSK3 in A. thaliana, to initiate GSK3-mediated inhibition of the MAPK pathway, leading to stomatal regulation [95]. The central components of this pathway are found in O. tauri; however, neither the upstream brassinosteroid signalling pathway nor the downstream stomatal regulation components are present. Similarly, in human and yeast cells MAPK cascades create complex signalling networks in a diverse array of processes [2,100], many of which are absent in O. tauri. Despite the diversity of processes, these central MAPK components from the CMGC and STE kinase families are among the most conserved protein kinase families in O. tauri (Additional file 7: Figure S5).
The residues of $\mathrm{MAP} 2 \mathrm{~K}$ that are proposed to be part of the GSK3 phosphorylation motif S/T-X-X-X-S/T are conserved in O. tauri, A. thaliana, S. cerevisiae and $H$. sapiens. The conserved residues are S182 and S186 in the O. tauri MAP2K (Ot04g04050). The residues S178 and S182 are conserved with those required for activation of MAP2K in human (MEK1) and yeast (STE7) [101]. The motif surrounding S178 appears to be more variable across eukaryotes than the downstream GSK3 motif. The residue corresponding to S178 is phosphorylated by a MAP3K (YODA) in A. thaliana [94]. Figure 7 shows the $O$. tauri components associated with the MAP2K pathway, along with a proposed schema based on the current $A$. thaliana model. The BSU family of green-lineage phosphatases, shown to regulate GSK3, are represented by a single member in O. tauri. The MAPK pathway, which GSK3 inhibits in A. thaliana, also has only a small number of possible components in O. tauri.

\section{Circadian signalling: CK1 and CK2}

Circadian rhythms are $\approx 24 \mathrm{~h}$ biological cycles, which arose as adaptations to daily changes in the environment. The circadian clock regulates diverse processes across eukaryotes, from the sleep-wake cycle of metazoa to photosynthesis [103]. O. tauri is already in use as a clock model for both in vivo and in silico studies $[25,30,104]$. In particular CK1 and CK2 have been shown to be part of conserved transcriptional/translational feedback loops in eukaryotes that regulate circadian clocks, based on pharmacological and overexpression results $[28,31,32]$.

\section{Casein Kinase 1 family}

The CK1 family of kinases are named after the highly conserved CK1 protein. CK1 has a variety of cellular functions, including regulation of membrane trafficking, DNA replication, Wnt signalling, RNA metabolism [105] and cell cycle regulation through tubulin binding [106,107]. CK1 isoforms have also been shown to affect circadian rhythmicity in metazoa $[108,109]$, in the fungus Neurospora crassa [110] and in O. tauri [31,32].

CK1 typically consists of a large number of paralogs per organism, a selection of which is shown in the phylogeny in Figure 8A (Additional file 7: Figure S5F). The human kinome contains seven isoforms $(C K 1 \alpha-\varepsilon)$, of 


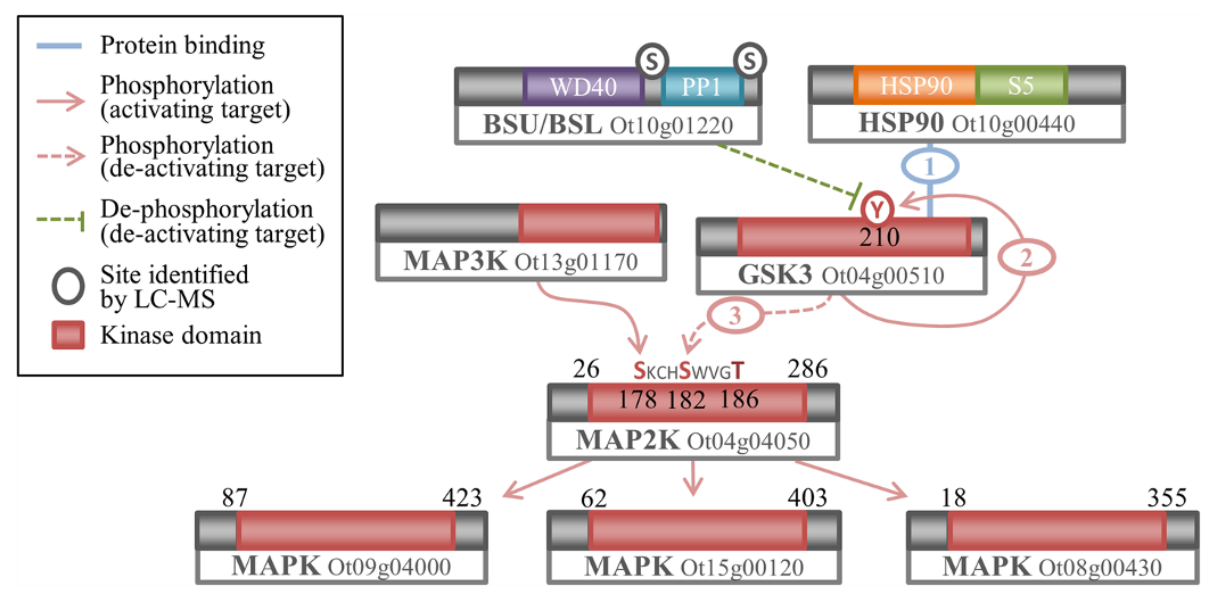

Figure 7 Schematic of GSK3 mediated signalling through the MAPK cascade in $\mathbf{O}$. tauri. BSU has been shown to dephosphorylate a $Y$ to inactivate GSK3 in plants [102], which is conserved as Y210 in GSK3. HSP90, which is conserved across eukaryotes has been shown to chaperone the autophosphorylation of the activating Y210 in GSK3 [59]. Conservation of S182 and T186 in the O. tauri MAP2K form part of a GSK3 phosphorylation motif, which when phosphorylated inhibit MAP2K activity in A. thaliana [94]. The upstream S182 is a conserved residue required for activation by MAP3K.

which the four least divergent are shown. The H. sapiens $\mathrm{CK} 1 \delta$ and $\mathrm{CK} 1 \varepsilon$ form the first branch after the divergence of $S$. cerevisiae, with a bootstrap confidence of 99\%. A. thaliana contains many CK1 paralogs, of which CKL1-13 are the most conserved. The closest $A$. thaliana CK1 has a distance of 0.23 to the point at which $H$. sapiens and S. cerevisiae diverge from the other sequences (Figure 8A). S. cerevisiae encodes three CK1 isoforms, of which the closest (HRR25, with a distance of 0.42) is included in Figure 8A. The O. tauri CK1 sequence (Ot02g06160) branches near the midpoint between $A$. thaliana and the $S$. cerevisiae and $H$. sapiens sequences, only a relatively short distance of 0.2 away from the $S$. cerevisiae and $H$. sapiens branch. O. tauri contains four further CK1-like protein kinases, of which only one (Ot02g06100) is conserved in A. thaliana (Additional file 7: Figure S5E).

Based on taxonomic studies of eukaryotes [21,111] we generally expect the Chlorophyta branch of algae and plant proteins to be more closely related, with a smaller distance between sequences from metazoa and yeast. However, our data shows that the distance of the O. tauri CK1 to the base of the branch that contains $H$. sapiens sequences is less than half the distance of $S$. cerevisiae to this branch. A. thaliana CK1 sequences also appear to have diverged considerably. The O. tauri CK1 is the closest among these three model organisms to human CK1 $\delta$ and CK1ع. This indicates that $O$. tauri may be an interesting model organism to study CK1.

\section{Casein Kinase 2 (CK2)}

Casein Kinase 2 (CK2) is a highly conserved kinase, found across all eukaryotes. CK2 is centrally important in many signalling pathways and is one of the most ubiquitous kinases in terms of substrate phosphorylation [112]. CK2 is a tetramer composed of a CK2 $\beta$ dimer and two $\mathrm{CK} 2 \alpha$ subunits. O. tauri contains a single catalytic CK $2 \alpha$ (Ot12g02620) and regulatory CK2 $\beta$ (Ot02g03010) subunit. The topology of the phylogenies for both subunits is very similar, the O. tauri CK2 appears to be more similar to the A. thaliana, D. melanogaster and $H$. sapiens than $S$. cerevisiae sequences. S. cerevisiae CK2 subunits have diverged considerably, similar to CK1, indicating that $O$. tauri may be interesting alternative model species for CK2.

\section{CK1, CK2 and the circadian clock}

CK2 is one of the few conserved components of the eukaryotic circadian clock $[28,103,113,114]$, where it fine-tunes period length and amplitude by dynamic modification of core-clock proteins. In A. thaliana, CK2 phosphorylates Circadian Clock-Associated 1(CCA1) and Late Elongated Hypocotyl (LHY) and over-expression of the CK2 regulatory subunit CKB3 shortens the period of these clock genes, accelerating plant flowering time $[115,116]$. CK2 is involved in temperature compensation of the clock in fungi and plants, which allows for robust timekeeping $[117,118]$. Within O. tauri we have identified CK2 motifs in CCA1 which are conserved (Figure 9, Additional file 4: Figure S3E) with the observed A. thaliana sites $[115,116]$, which indicate that this interaction may be retained. We also observed an S109 phosphorylation, in a Chlorophyta specific region, at the C-terminus of the MYB DNA binding domain which conforms to an E-X-S CK2 substrate motif. In metazoa, CK2 $\alpha$ directly phosphorylates PERIOD (PER). Mutation of CK2 delays the nuclear import of PER and lengthens circadian period [119] (Figure 9). A mutation in CK2 $\beta$ 


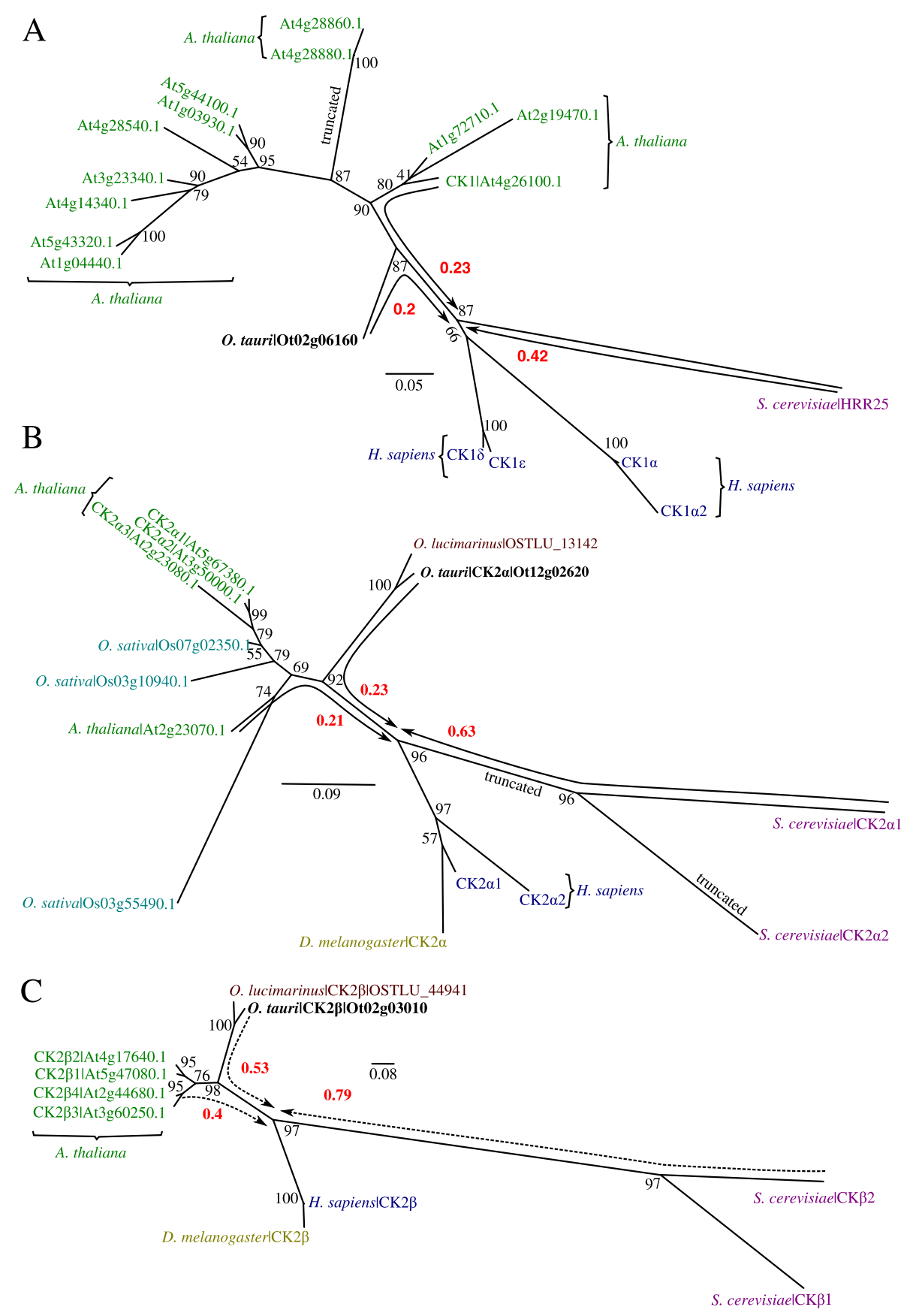

Figure 8 Phylogenies CK1, CK2a and CK2 $\beta$. (A) Phylogeny of CK1, revealing an O. tauri ortholog in close proximity $H$. sapiens (relative to A. thaliana and S. cerevisiae). (B) Phylogeny of CK2a catalytic subunit showing the closer proximity of $A$. thaliana CK2a to the $H$. sapiens sequences (relative to $S$. cerevisiae). (C) Phylogeny of CK2 $\beta$ regulatory subunit, revealing a similar topology and rate of relative divergence to the CK2a subunit. A general explanation for phylogenies is in Figure 2. Raw branch lengths (red) are annotated to broken-line arrows and show distances from O. tauri, A. thaliana, and S. cerevisiae to the H. sapiens and D. melanogaster divergence point. For (B) the H. sapiens CK1Y isoforms are not shown, as they are more divergent than the other sequences included. The CK2a2 branch has been truncated as it was considerably more divergent than CK2a1. S. cerevisiae identifiers are standard names from SGD. Accessions for D. melanogaster and H. sapiens proteins are given in Additional file 9: Table S3.

also lengthens period [120]. CK2 regulates the mammalian clock by binding and phosphorylating the clock protein PER2 at S/T residues in the $\mathrm{N}$-terminus, thereby stabilising the protein and promoting nuclear accumulation of PER2 [121]. Diminished PER2 stability can have opposing effects on the circadian period by affecting the 

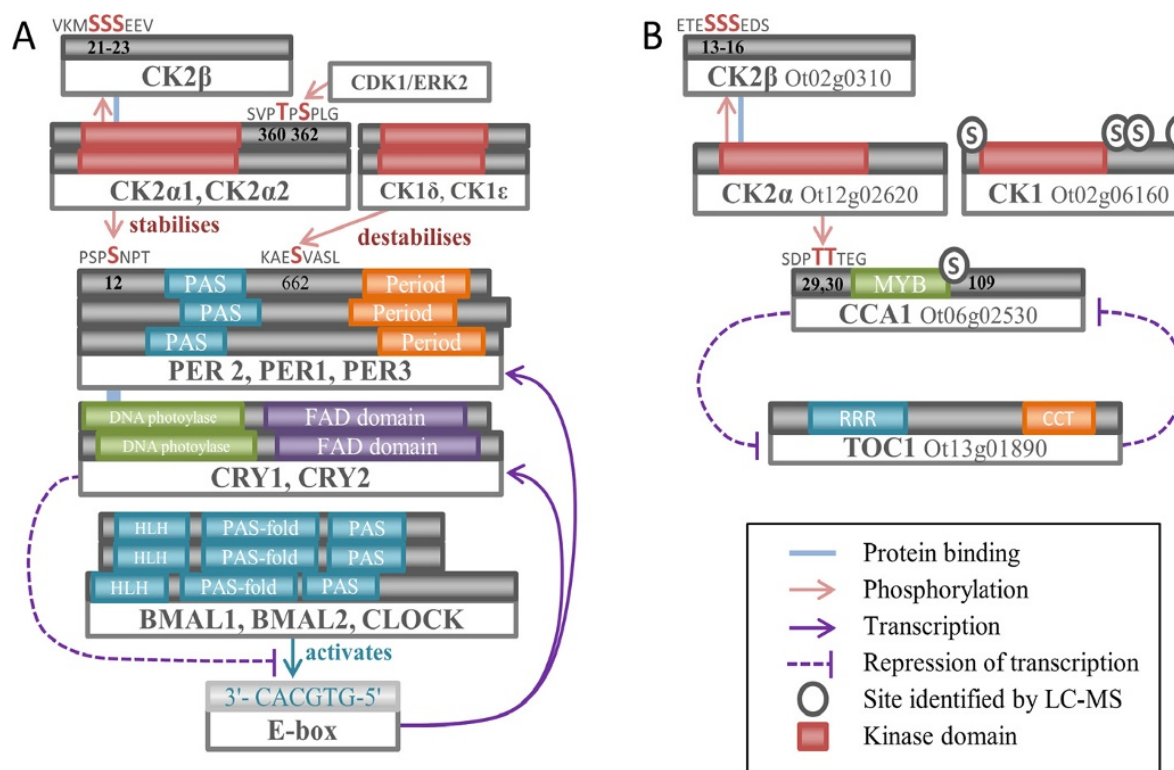

Figure 9 The core transcriptional circadian clock of (A) H. sapiens and (B) O. tauri. CK1 and CK2 regulate both clocks though PER and CCA1 phosphorylation. The downstream core clock however differs: in H. sapiens, CK2 has been shown to stabilise PER2 and promote its nuclear accumulation [121]. PER2 in turn stabilises Cryptochrome (CRY)1/2 in complex, which represses the transcriptional activation of PER1/2/3 via CLOCK/BMAL1/2, creating an oscillating feedback loop. In O. tauri, CK2 phosphorylates the morning-expressed CCA1 (by similarity with A. thaliana, which represses the transcription of the evening-expressed TOC1. TOC1 in return represses the transcription of CCA1, again creating an oscillating feedback loop. PAS - Period - FAD - HLH - helix-loop-helix domain MYB - RRR - response regulator receiver domain CCT - CCT motif.

timing of when PER2 is accumulated in the nucleus accelerated and prolonged nuclear import speeds up and slows down the clock, respectively.

A naturally-occurring short circadian clock period phenotype of 20 hours was first observed in Syrian hamsters (Mesocricetus auratus) and attributed to the tau mutation in CK1ع [122]. The tau mutation increases PER1 and PER2 phosphorylation, increasing proteasomal degradation, and shortening the circadian period [123]. CK1 control of the clock is exemplified by familial advanced sleep phase syndrome, a condition associated with early sleep time followed by early morning awakening, whereby a mutation to human PER2 or CK1 $\delta$ advances period [124-126]. Mutations to D. melanogaster CK1 $\delta$ lengthen period, suggesting differences in the regulation between mammalian and insect clocks [126]. Until recently, CK1 has not been implicated in plant clocks. There are early indications for a functional role for CK1 in the O. tauri clock [31,32], however the exact targets of CK1 are unknown.

\section{Smaller kinase families in 0 . tauri}

Additional file 5: Table S2 shows that the remaining protein kinases span a wide range of families, which are discussed in Additional file 8: Figure S6: two of the highly-conserved RIO family, a Polo-like kinase (PLK), an Aurora kinase, BUD32, five STN-like kinases, two BUB1like kinases, Haspin, and two HKs. The O. tauri kinome therefore comprises a suitably diverse set to represent many of the known protein kinase functions in eukaryotes.

\section{Conclusion}

We identified 133 gene loci encoding catalytic protein kinases in O. tauri, constituting a small kinome of a similar order to $S$. cerevisiae (130 genes). As a photosynthetic model for core signalling, it is nearly ten-fold smaller than the $A$. thaliana kinome [4]. Comparing A. thaliana, S. cerevisiae and $H$. sapiens sequences, we found $O$. tauri kinases were frequently more closely related to the $H$. sapiens sequences than were the $S$. cerevisiae orthologs (the PIKK kinases are one exception). Thus genome reduction in O. tauri has not led to divergence in its kinome, in contrast to the minimal kinomes of parasitic species. DNA-PK is present in O. tauri and $H$. sapiens but absent in A. thaliana; PKG in O. tauri is also closer to the metazoan and fungal sequences than the closest $A$. thaliana sequences; several other components are conserved in exemplar signalling pathways (genes, phosphorylated amino acids and binding motifs), such as S6K activation via the TORC1 pathway. Together with other conserved components that are otherwise absent or poorly conserved in much of the green lineage, such as the cell cycle phosphatase CDC25 [127], our kinome survey indicates that $O$. tauri is a reduced but representative laboratory model species for signalling 
research, which incorporates many eukaryote-wide signalling components.

\section{Methods}

\section{Identification of OrthoMCL ortholog-groups}

We obtained ortholog groups assignment for $H$. sapiens, S. cerevisiae and $A$. thaliana from the OrthoMCL version 5 database and used the proteome upload service [128] to annotate the $O$. tauri peptide sequence from BEG (Additional file 3: Figure S2), which we supplemented with our corrected gene models.

\section{Identification of kinases in O. tauri}

In an approach similar to Vilella et al. [129] we clustered all sequences from $H$. sapiens, S. cerevisiae, A. thaliana, $O$. lucimarinus, and O. tauri into related protein families. For O. tauri we used the December 2006 peptides sequences, and for O. lucimarinus we used the JGI November 2011 peptide sequences, both retrieved from BEG (Additional file 3: Figure S2). The TAIR (version 10) representative gene models for A. thaliana and Uniprot reference proteomes for S. cerevisiae and $H$. sapiens were retrieved in November 2012. We searched all proteins, against all proteins, using the NCBI blastp tool (version 2.2.25; BLOSUM62) with an e-value cut-off of 0.01. We calculated the BLAST Ratio Score (BSR) for each hit found [130] and we retained best-reciprocal hits and BSR scores greater than $1 / 3$. We created a distance matrix using the BSR scores and applied the Markov Cluster (MCL) algorithm, (version 12-068), with inflation values 1.1 and 1.4. We extracted groups of kinases and phosphatases from the subsequent clusters. We searched for kinase and phosphatase catalytic domains with the hmmsearch algorithm (HMMER 3.0, GA cut-off) [131] using the models provided in Pfam-A (January 2013) [132]. We also ran a sequence similarity search with an e-value cut-off of 0.07 , using the blastp algorithm (BLOSUM62), from O. tauri against the PlantsP database. All these results were manually curated to extract all candidate kinases and phosphatases into a database.

\section{Constructing alignments and phylogenies}

Kinase alignments for each family in $O$. tauri were constructed by whole sequence alignment of protein sequences to whole families of proteins. The KinBase database was used as a source of $S$. cerevisiae [133] and $H$. sapiens [6] kinases annotations (Additional file 9) and family. The PlantsP [37] database provided A. thaliana kinase annotations. We aligned sequences using MAFFT [134] version 6 within JalView [135,136]. We used the high quality global alignment algorithm G-INS-i, with BLOSUM62, 2-tree rebuilds, gap open and extension penalties of 1.53 and 0.12 respectively, and a limit of 1,000 iterations. Poorly aligned sequences were manually removed from the alignment. For editing alignments of more than 8 sequences we used guidance version 1.3.1, with the same MAFFT parameters previously described, and 100 guidance bootstraps [137]. We retained columns with a confidence value greater than 0.93 , and sequences with a confidence value above 0.6. Columns with gaps were excluded. Inference of phylogenetic trees on the conserved alignment columns was performed using a Maximum Likelihood (ML) approach. Phylogenies were built with RaXML version 7.2.8 [138]. We used a $\gamma$ model of evolutionary rate heterogeneity combined with an estimation of the proportion of invariant sites. Amino acid replacement scoring was determined using the WAG matrix [139]. Support for branches on the ML tree was evaluated using bootstrap analysis, using the frequency-based criteria (FC) parameter to determine the number of iterations. We used the FigTree version 1.4.0 tool for the visualisation of trees.

When alignments of $O$. tauri proteins contained gaps, extended inserts which were not found in other species, or poor alignments, we investigated and where appropriate corrected underlying gene models. Where gaps where present in the $O$. tauri genomic sequence, we used the closest gene from $O$. lucimarinus to infer the gap sequence, when there was a high degree of conservation in the adjacent region (Additional file 3: Figure S2).

\section{Phosphorylation-site identification by tandem mass spectrometry}

Protein extract from $O$. tauri cells was prepared in a similar manner as described previously [26], with the digestion performed on $300 \mu \mathrm{g}$ protein extract. Peptides were cleaned by reverse phase and phosphopeptide enrichment and LC-MS analysis were performed as described previously [26].

All multi-charged ions $(2+, 3+, 4+)$ were extracted from each LC-MS file and MSMS data was searched using MASCOT Version 2.4 (Matrix Science Ltd, UK) against the $O$. tauri subset of the NCBI protein database $(12 / 01 / 2011 ; 8,726$ sequences) using a maximum missedcut value of 2, variable oxidation (M), N-terminal protein acetylation, phosphorylation (S, T, and $\mathrm{Y}$ ) and fixed carbamidomethylation (C). Precursor mass tolerance was $7 \mathrm{ppm}$ and MSMS tolerance $0.4 \mathrm{amu}$. The significance threshold (p) was set below 0.05 (MudPIT scoring). A minimum peptide cut off score of 20 was set, corresponding to $<3 \%$ global false discovery rate (FDR) using a decoy database search.

Ambiguous sites were confirmed by cross-referencing (by sequence, charge, and quantity of residue modifications) with most probable site predictions from MaxQuant (version 1.0.13.8 in singlet mode, same Mascot settings) [140]. 


\section{Availability of supporting data}

All sequences and supporting data are included as additional files and are available at http://hdl.handle. net/10283/563.

\section{Additional files}

Additional file 1: Table S1. Phosphorylated peptides identified by LC-MS. A compilation of 3,994 uniquely identified phosphorylation sites in 0 . tauri. These describe phosphorylations of 2,214 peptide sequences, which correspond to 1,252 proteins.

Additional file 2: Figure S1. Protein domain diagrams with phospho-sites. Domain diagrams for $O$. tauri protein kinases, grouped according to protein family. PfamA protein domains were detected by hmmr3 (PfamA downloaded Oct-2013). The locations of experimentally observed phosphorylation sites are annotated above the domain track.

Additional file 3: Figure S2. Sequences for novel and adjusted $O$. tauri gene models. We list the default sources of protein sequences and 0 . tauri sequences that were novel, derived from adjusted gene models, or have been patched with orthologous sequence from 0 . lucimarinus.

Additional file 4: Figure S3. Evidence for conservation of phospho-site regions in $\mathrm{O}$ tauri. Sequence alignments for conserved phospho-site regions implicated in the phosphorylation of (A) MAP2K (Ot04g04050) by MAP3K and GSK3, (B) S6K (Ot07g02590) by GSK3, (C) S6K (Ot07g02590) by TORC1, (D) S6K (Ot07g02590) by PDK1 and (E) CCA1 (Ot06g02530) by CK2. Black framed amino acids specify a conserved phosphorylation site, and red framed residues show the conservation of a pre-primed phosphorylation site.

Additional file 5: Table S2. Kinase and phosphatase classifications in O. tauri. The description and classification of $133 \mathrm{O}$. tauri protein kinases and 34 phosphatases based on sequence phylogeny and domain structure.

Additional file 6: Figure S4. Kinase similarities between O. tauri and other model organisms. Box and whiskers plot that describes for each species the distributions of the Blast Score Ratio (BSR) similarities of $O$. tauri protein kinases against the best hit in the given species. BSR scores for each $O$. tauri kinase are calculated against the best hit (highest score) found in H. sapiens, S. cerevisiae, A. thaliana, and O. lucimarinus. Red crosses show the outliers, whiskers indicate the extremes of the distribution (excluding outliers). Boxes show the upper and lower quartiles, dissected by the red median line. The blue dot indicates the mean. Notches, indicating the $95 \%$ confidence interval, were calculated from 100,000 bootstraps. Table one of Additional file 6 gives the significance of the differences between species for the mean Blast Score Ratio (BSR) similarities of kinases.

Additional file 7: Figure S5. Phylogenies of the main kinase families. Phylogenies for the (A) CMGC, (B) AGC, (C) CAMK, (D) STE, and (E) CK1 protein kinase families. H. sapiens and S. cerevisiae kinases are labelled according to their KinBase identifiers. A. thaliana kinases are labelled with AGl accessions. Accessions for $O$. tauri sequences refer to the BEG gene models except where we have altered a gene model (Additional file 2: Figure S2). Bootstrap confidences are assigned to edges. A broken-line edge indicates a bootstrap confidence of less than 40 .

Additional file 8: Figure S6. Discussion and phylogenies of further kinase families. Phylogenies and descriptions for the (A) TKL, (B) BUD32, (C) RIO, (D) STN, (E) SCYL (F) ABC1, and (G) Histidine Kinases families.

Additional file 9: Table S3. Sequence accessions for phylogenies RefSeq and UniProt protein sequence accessions for the H. sapiens and D. melanogaster sequences, referred to by gene name in the phylogenies.

\section{Competing interests}

The authors declare that they have no competing interests.

\section{Authors' contributions}

$\mathrm{MMH}, \mathrm{SFM}, \mathrm{GVO}, \mathrm{TIS}, \mathrm{TLB}$ and AJM were involved in the conception and structure of the work. MMH conducted the bioinformatics analysis. MEBL, SFM and TLB generated and analysed the phosphorylation dataset from
O. tauri. MMH, SFM and ZBN wrote the paper, with contributions from all authors. All authors read and approved the final manuscript.

\section{Acknowledgements}

We are grateful to Bram Verhelst from VIB Department of Plant Systems Biology, Ghent University, for advice concerning O. tauri gene models. GvO is a Royal Society University Research Fellow (UF110173) supported by Royal Society research grant RG120372. This work was supported by BBSRC and EPSRC awards BB/D019621 and BB/J009423.

\section{Author details}

'SynthSys and School of Biological Sciences, University of Edinburgh, Edinburgh EH9 3JD, UK. ${ }^{2}$ Institute of Structural and Molecular Biology, University of Edinburgh, Edinburgh EH9 3JR, UK. ${ }^{3}$ Biomathematics \& Statistics Scotland, University of Edinburgh, Edinburgh EH9 3JZ, UK. ${ }^{4}$ nstitute for Adaptive and Neural Computation, School of Informatics, University of Edinburgh, Edinburgh EH8 9AB, UK

Received: 24 January 2014 Accepted: 8 July 2014

Published: 2 August 2014

\section{References}

1. Hanks SK, Hunter T: Protein kinases 6. The eukaryotic protein kinase superfamily: kinase (catalytic) domain structure and classification. FASEB $J$ 1995, 9:576-596

2. Keshet Y, Seger R: The MAP Kinase Signaling Cascades: A System of Hundreds of Components Regulates a Diverse Array of Physiological Functions. In MAP Kinase Signaling Protocols. Methods in Molecular Biology, vol. 661. Edited by Seger R. Totowa: Humana Press; 2010:3-38.

3. Breitkreutz A, Choi H, Sharom JR, Boucher L, Neduva V, Larsen B, Lin Z-Y, Breitkreutz B-J, Stark C, Liu G, Ahn J, Dewar-Darch D, Reguly T, Tang X, Almeida R, Qin ZS, Pawson T, Gingras A-C, Nesvizhskii Al, Tyers M: A global protein kinase and phosphatase interaction network in yeast. Science 2010, 328:1043-1046.

4. Champion A, Kreis M, Mockaitis K, Picaud A, Henry Y: Arabidopsis kinome: after the casting. Funct Integr Genomics 2004, 4:163-187.

5. Goldberg JM, Manning G, Liu A, Fey P, Pilcher KE, Xu Y, Smith JL: The Dictyostelium kinome-analysis of the protein kinases from a simple model organism. PLOS Genet 2006, 2:e38.

6. Manning G, Plowman GD, Hunter T, Sudarsanam S: Evolution of protein kinase signaling from yeast to man. Trends Biochem Sci 2002, 27:514-520.

7. Miranda-Saavedra D, Stark MJR, Packer JC, Vivares CP, Doerig C, Barton GJ: The complement of protein kinases of the microsporidium Encephalitozoon cuniculi in relation to those of Saccharomyces cerevisiae and Schizosaccharomyces pombe. BMC Genomics 2007, 8:309.

8. Manning G, Reiner DS, Lauwaet T, Dacre M, Smith A, Zhai Y, Svard S, Gillin FD: The minimal kinome of Giardia lamblia illuminates early kinase evolution and unique parasite biology. Genome Biol 2011, 12:R66.

9. Deutscher J, Saier MH Jr: Ser/Thr/Tyr protein phosphorylation in bacteria for long time neglected, now well established. J Mol Microbiol Biotechnol 2005, 9:125-131.

10. Kennelly PJ: Protein Ser/Thr/Tyr phosphorylation in the archaea. J Biol Chem 2014, 289:9480-9487.

11. Kannan N, Taylor SS, Zhai Y, Venter JC, Manning G: Structural and functional diversity of the microbial kinome. PLOS Biol 2007, 5:e17.

12. Scheeff ED, Bourne PE: Structural evolution of the protein kinase-like superfamily. PLoS Comput Biol 2005, 1:e49.

13. Kumar MN, Jane W-N, Verslues PE: Role of the putative osmosensor Arabidopsis histidine kinase 1 in dehydration avoidance and lowwater-potential response. Plant Physiol 2013, 161:942-953.

14. Hall BP, Shakeel SN, Amir M, UI Haq N, Qu X, Schaller GE: Histidine kinase activity of the ethylene receptor ETR1 facilitates the ethylene response in Arabidopsis. Plant Physiol 2012, 159:682-695.

15. Hanks SK, Quinn AM, Hunter T: The protein kinase family: conserved features and deduced phylogeny of the catalytic domains. Science 1988 241:42-52.

16. Leonard CJ, Aravind L, Koonin EV: Novel families of putative protein kinases in bacteria and archaea: evolution of the "eukaryotic" protein kinase superfamily. Genome Res 1998, 8:1038-1047.

17. Brinkworth Rl, Munn AL, Kobe B: Protein kinases associated with the yeast phosphoproteome. BMC Bioinformatics 2006, 7:47. 
18. Ghelis T: Signal processing by protein tyrosine phosphorylation in plants. Plant Signal Behav 2011, 6:942-951.

19. Courties C, Vaquer A, Troussellier M, Lautier J, Chrétiennot-Dinet MJ, Neveux J, Machado C, Claustre H: Smallest eukaryotic organism. Nature 1994, 370:255-255.

20. Palenik B, Grimwood J, Aerts A, Rouzé P, Salamov A, Putnam N, Dupont C, Jorgensen R, Derelle E, Rombauts S, Zhou K, Otillar R, Merchant SS, Podell S, Gaasterland T, Napoli C, Gendler K, Manuell A, Tai V, Vallon O, Piganeau G, Jancek S, Heijde M, Jabbari K, Bowler C, Lohr M, Robbens S, Werner G, Dubchak I, Pazour GJ, et al: The tiny eukaryote Ostreococcus provides genomic insights into the paradox of plankton speciation. Proc Natl Acad Sci U S A 2007, 104:7705-7710.

21. Keeling PJ, Burger G, Durnford DG, Lang BF, Lee RW, Pearlman RE, Roger AJ, Gray MW: The tree of eukaryotes. Trends Ecol Evol 2005, 20:670-676.

22. Henderson GP, Gan L, Jensen GJ: 3-D ultrastructure of O. tauri: electron cryotomography of an entire eukaryotic cell. PLOS One 2007, 2:e749.

23. Farinas B, Mary C, De Manes C-L O, Bhaud Y, Peaucellier G, Moreau H: Natural synchronisation for the study of cell division in the green unicellular alga Ostreococcus tauri. Plant Mol Biol 2006, 60:277-292.

24. van Ooijen G, Knox K, Kis K, Bouget F-Y, Millar AJ: Genomic transformation of the picoeukaryote Ostreococcus tauri. J Vis Exp 2012, (65):e4074.

25. Corellou F, Schwartz C, Motta J-P, Djouani-Tahri EB, Sanchez F, Bouget F-Y: Clocks in the green lineage: comparative functional analysis of the circadian architecture of the picoeukaryote ostreococcus. Plant Cell 2009, 21:3436-3449

26. Le Bihan T, Martin SF, Chirnside ES, van Ooijen G, Barrios-Llerena ME, O'Neill JS, Shliaha PV, Kerr LE, Millar AJ: Shotgun proteomic analysis of the unicellular alga Ostreococcus tauri. J Proteomics 2011, 74:2060-2070.

27. Martin SF, Munagapati VS, Salvo-Chirnside E, Kerr LE, Le Bihan T: Proteome turnover in the green alga Ostreococcus tauri by time course $15 \mathrm{~N}$ metabolic labeling mass spectrometry. J Proteome Res 2012, 11:476-486.

28. O'Neill JS, van Ooijen G, Dixon LE, Troein C, Corellou F, Bouget F-Y, Reddy $\mathrm{AB}$, Millar AJ: Circadian rhythms persist without transcription in a eukaryote. Nature 2011, 469:554-558.

29. van Ooijen G, Dixon LE, Troein C, Millar AJ: Proteasome function is required for biological timing throughout the twenty-four hour cycle. Curr Biol 2011, 21:869-875.

30. Troein C, Corellou F, Dixon LE, van Ooijen G, O'Neill JS, Bouget F-Y, Millar AJ: Multiple light inputs to a simple clock circuit allow complex biological rhythms. Plant J 2011, 66:375-385.

31. van Ooijen G, Hindle M, Martin SF, Barrios-Llerena M, Sanchez F, Bouget F-Y, O'Neill JS, Bihan TL, Millar AJ: Functional analysis of casein kinase 1 in a minimal circadian system. PLoS One 2013, 8:e70021.

32. van Ooijen G, Martin SF, Barrios-Llerena ME, Hindle M, Le Bihan T, O'Neill JS, Millar AJ: Functional analysis of the rodent CK1tau mutation in the circadian clock of a marine unicellular alga. BMC Cell Biol 2013, 14:46.

33. Chen F, Mackey AJ, Stoeckert CJ, Roos DS: OrthoMCL-DB: querying a comprehensive multi-species collection of ortholog groups. Nucl Acids Res 2006, 34(suppl 1):D363-D368.

34. Shiu S-H, Bleecker AB: Receptor-like kinases from Arabidopsis form a monophyletic gene family related to animal receptor kinases. PNAS 2001, 98:10763-10768

35. Miranda-Saavedra D, Barton GJ: Classification and functional annotation of eukaryotic protein kinases. Proteins Struct Funct Bioinformatics 2007, 68:893-914.

36. Manning $G$, Whyte DB, Martinez R, Hunter T, Sudarsanam S: The protein kinase complement of the human genome. Science 2002, 298:1912-1934.

37. Tchieu JH, Fana F, Fink JL, Harper J, Nair TM, Niedner RH, Smith DW, Steube K, Tam TM, Veretnik S, Wang D, Gribskov M: The plantsP and plantsT functional genomics databases. Nucleic Acids Res 2003, 31:342-344.

38. Liberti S, Sacco F, Calderone A, Perfetto L, lannuccelli M, Panni S, Santonico E, Palma A, Nardozza AP, Castagnoli L, Cesareni G: HuPho: the human phosphatase portal. FEBS J 2013, 280:379-387.

39. Lehti-Shiu MD, Zou C, Hanada K, Shiu S-H: Evolutionary history and stress regulation of plant receptor-like kinase/pelle genes. Plant Physiol 2009, 150:12-26.

40. Lehti-Shiu MD, Shiu S-H: Diversity, classification and function of the plant protein kinase superfamily. Phil Trans R Soc B 2012, 367:2619-2639.

41. Kosti I, Mandel-Gutfreund Y, Glaser F, Horwitz BA: Comparative analysis of fungal protein kinases and associated domains. BMC Genomics 2010, 11:133.
42. Christie JM, Yang H, Richter GL, Sullivan S, Thomson CE, Lin J, Titapiwatanakun B, Ennis M, Kaiserli E, Lee OR, Adamec J, Peer WA, Murphy AS: Phot1 inhibition of $A B C B 19$ primes lateral auxin fluxes in the shoot apex required for phototropism. PLOS Biol 2011, 9:e1001076.

43. Gao Q, Yang Z, Zhou Y, Yin Z, Qiu J, Liang G, Xu C: Characterization of an $A b c 1$ kinase family gene OsABC1-2 conferring enhanced tolerance to dark-induced stress in rice. Gene 2012, 498:155-163.

44. Yang S, Zeng X, Li T, Liu M, Zhang S, Gao S, Wang Y, Peng C, Li L, Yang C: AtACDO1, an ABC1-like kinase gene, is involved in chlorophyll degradation and the response to photooxidative stress in Arabidopsis. J Exp Bot 2012, 63:3959-3973

45. Yang S: AtSIA1, an ABC1-like kinase, regulates salt response in Arabidopsis. Biologia 2012, 67:1107-1111.

46. Lozano J-C, Schatt P, Botebol H, Vergé V, Lesuisse E, Blain S, Carré IA, Bouget F-Y: Efficient gene targeting and foreign DNA removal by homologous recombination in the picoeukaryote Ostreococcus. Plant J 2014, 76:6.

47. van Dam TJP, Zwartkruis FJT, Bos JL, Snel B: Evolution of the TOR pathway. J Mol Evol 2011, 73:209-220.

48. Shin S, Wolgamott L, Yu Y, Blenis J, Yoon S-O: Glycogen synthase kinase (GSK)-3 promotes p70 ribosomal protein S6 kinase (p70S6K) activity and cell proliferation. PNAS 2011, 108:E1204-E1213.

49. Warmerdam DO, Kanaar R: Dealing with DNA damage: relationships between checkpoint and repair pathways. Mutat Res 2010, 704:2-11.

50. Templeton GW, Moorhead GBG: The phosphoinositide-3-OH-kinase-related kinases of Arabidopsis thaliana. EMBO Rep 2005, 6:723-728.

51. Lloyd JPB, Davies B: SMG1 is an ancient nonsense-mediated mRNA decay effector. Plant J 2013, 76:800-810.

52. Lieber MR, Ma Y, Pannicke U, Schwarz K: Mechanism and regulation of human non-homologous DNA end-joining. Nat Rev Mol Cell Biol 2003, 4:712-720.

53. Liu S, Opiyo SO, Manthey K, Glanzer JG, Ashley AK, Amerin C, Troksa K, Shrivastav M, Nickoloff JA, Oakley GG: Distinct roles for DNA-PK, ATM and ATR in RPA phosphorylation and checkpoint activation in response to replication stress. Nucl Acids Res 2012, 40:10780-10794.

54. Marwedel T, Ishibashi T, Lorbiecke R, Jacob S, Sakaguchi K, Sauter M: Plantspecific regulation of replication protein A2 (OsRPA2) from rice during the cell cycle and in response to ultraviolet light exposure. Planta 2003, 217:457-465.

55. Ferguson BJ, Mansur DS, Peters NE, Ren H, Smith GL: DNA-PK is a DNA sensor for IRF-3-dependent innate immunity. Elife 2012, 1:e00047.

56. Robbens $S$, Khadaroo B, Camasses A, Derelle E, Ferraz C, Inzé D, van de Peer Y, Moreau H: Genome-wide analysis of core cell cycle genes in the unicellular green alga Ostreococcus tauri. Mol Biol Evol 2005, 22:589-597.

57. CristinaRodriguez $\mathrm{M}$, Petersen $\mathrm{M}, \mathrm{Mundy} J$ : Mitogen-activated protein kinase signaling in plants. Annu Rev Plant Biol 2010, 61:621-649.

58. Seger R, Krebs EG: The MAPK signaling cascade. FASEB J 1995, 9:726-735

59. Lochhead PA, Kinstrie R, Sibbet G, Rawjee T, Morrice N, Cleghon V: A chaperone-dependent GSK3beta transitional intermediate mediates activation-loop autophosphorylation. Mol Cell 2006, 24:627-633.

60. Saidi Y, Hearn TJ, Coates JC: Function and evolution of "green" GSK3/ Shaggy-like kinases. Trends Plant Sci 2012, 17:39-46.

61. Krishna P, Gloor G: The Hsp90 family of proteins in Arabidopsis thaliana. Cell Stress Chaperones 2001, 6:238-246.

62. Dittrich ACN, Devarenne TP: Perspectives in PDK1 evolution. Plant Signal Behav 2012, 7:642-649.

63. Rademacher EH, Offringa R: Evolutionary adaptations of plant AGC kinases: from light signaling to cell polarity regulation. Front Plant SCi 2012, 3:250.

64. Mora A, Komander D, van Aalten DMF, Alessi DR: PDK1, the master regulator of AGC kinase signal transduction. Semin Cell Dev Biol 2004, 15:161-170.

65. Biondi RM, Kieloch A, Currie RA, Deak M, Alessi DR: The PIF-binding pocket in PDK1 is essential for activation of S6K and SGK, but not PKB. EMBO J 2001, 20:4380-4390.

66. Osborne BW, Wu J, McFarland CJ, Nickl CK, Sankaran B, Casteel DE, Woods VL, Kornev AP, Taylor SS, Dostmann WR: Crystal structure of cGMP-dependent protein kinase reveals novel site of interchain communication. Structure 2011, 19:1317-1327.

67. Taylor SS, llouz R, Zhang P, Kornev AP: Assembly of allosteric macromolecular switches: lessons from PKA. Nat Rev Mol Cell Biol 2012, 13:646-658. 
68. Zimmermann B, Chiorini JA, Ma Y, Kotin RM, Herberg FW: PrKX is a novel catalytic subunit of the CAMP-dependent protein kinase regulated by the regulatory subunit type I. J Biol Chem 1999, 274:5370-5378.

69. Hornbeck PV, Chabra I, Kornhauser JM, Skrzypek E, Zhang B: PhosphoSite: a bioinformatics resource dedicated to physiological protein phosphorylation. Proteomics 2004, 4:1551-1561.

70. Wullschleger S, Loewith R, Hall MN: TOR signaling in growth and metabolism. Cell 2006, 124:471-484.

71. Xiong $Y$, Sheen J: Rapamycin and glucose-target of rapamycin (TOR) protein signaling in plants. J Biol Chem 2012, 287:2836-2842.

72. Loewith R: 9 - TORC1 Signaling in Budding Yeast. In The Enzymes. Volume 27. Edited by Michael Hall N. Fuyuhiko Tamanoi: Academic Press; 2010:147-175.

73. Cybulski N, Hall MN: TOR complex 2: a signaling pathway of its own. Trends Biochem Sci 2009, 34:620-627.

74. Alessi DR, Kozlowski MT, Weng QP, Morrice N, Avruch J: 3-Phosphoinositidedependent protein kinase 1 (PDK1) phosphorylates and activates the p70 S6 kinase in vivo and in vitro. Curr Biol 1998, 8:69-81.

75. Pullen N, Dennis PB, Andjelkovic M, Dufner A, Kozma SC, Hemmings BA Thomas G: Phosphorylation and activation of p70s6k by PDK1. Science 1998, 279:707-710

76. Burnett PE, Barrow RK, Cohen NA, Snyder SH, Sabatini DM: RAFT1 phosphorylation of the translational regulators p70 S6 kinase and 4E-BP1. Proc Natl Acad Sci U S A 1998, 95:1432-1437.

77. Moser BA, Dennis PB, Pullen N, Pearson RB, Williamson NA, Wettenhall RE, Kozma SC, Thomas G: Dual requirement for a newly identified phosphorylation site in p70s6k. Mol Cell Biol 1997, 17:5648-5655.

78. Casamayor A, Morrice NA, Alessi DR: Phosphorylation of Ser-241 is essential for the activity of 3-phosphoinositide-dependent protein kinase-1: identification of five sites of phosphorylation in vivo. Biochem J 1999, 342(Pt 2):287-292.

79. Sato S, Fujita N, Tsuruo T: Regulation of kinase activity of 3-phosphoinositidedependent protein kinase-1 by binding to 14-3-3. J Biol Chem 2002, 277:39360-39367.

80. Otterhag L, Gustavsson N, Alsterfjord M, Pical C, Lehrach H, Gobom J, Sommarin M: Arabidopsis PDK1: identification of sites important for activity and downstream phosphorylation of S6 kinase. Biochimie 2006, 88:11-21.

81. Jiang $Y$, Broach JR: Tor proteins and protein phosphatase $2 A$ reciprocally regulate Tap42 in controlling cell growth in yeast. EMBO J 1999, 18:2782-2792.

82. Peterson RT, Desai BN, Hardwick JS, Schreiber SL: Protein phosphatase 2A interacts with the 70-kDa S6 kinase and is activated by inhibition of FKBP12-rapamycinassociated protein. PNAS 1999, 96:4438-4442.

83. Cygnar KD, Gao X, Pan D, Neufeld TP: The phosphatase subunit tap42 functions independently of target of rapamycin to regulate cell division and survival in Drosophila. Genetics 2005, 170:733-740.

84. Hrabak EM, Chan CWM, Gribskov M, Harper JF, Choi JH, Halford N, Kudla J, Luan S, Nimmo HG, Sussman MR, Thomas M, Walker-Simmons K, Zhu J-K, Harmon AC: The Arabidopsis CDPK-SnRK superfamily of protein kinases. Plant Physiol 2003, 132:666-680

85. Klimecka M, Muszyńska G: Structure and functions of plant calciumdependent protein kinases. Acta Biochim Pol 2007, 54:219-233.

86. Ghillebert R, Swinnen E, Wen J, Vandesteene L, Ramon M, Norga K, Rolland F, Winderickx J: The AMPK/SNF1/SnRK1 fuel gauge and energy regulator: structure, function and regulation. FEBS J 2011, 278:3978-3990

87. Usaite R, Jewett MC, Oliveira AP, Yates JR, Olsson L, Nielsen J: Reconstruction of the yeast Snf1 kinase regulatory network reveals its role as a global energy regulator. Mol Syst Biol 2009, 5:319.

88. Polge C, Thomas M: SNF1/AMPK/SnRK1 kinases, global regulators at the heart of energy control? Trends Plant Sci 2007, 12:20-28.

89. Hamel L-P, Sheen J, Séguin A: Ancient signals: comparative genomics of green plant CDPKs. Trends Plant Sci 2013, 19:79-89.

90. Kanchiswamy CN, Takahashi H, Quadro S, Maffei ME, Bossi S, Bertea C, Zebelo SA, Muroi A, Ishihama N, Yoshioka H, Boland W, Takabayashi J, Endo Y, Sawasaki T, Arimura G: Regulation of Arabidopsis defense responses against Spodoptera littoralis by CPK-mediated calcium signaling. BMC Plant Biology 2010, 10:97.

91. Mori IC, Murata Y, Yang Y, Munemasa S, Wang Y-F, Andreoli S, Tiriac H, Alonso JM, Harper JF, Ecker JR, Kwak JM, Schroeder JI: CDPKs CPK6 and
CPK3 function in ABA regulation of guard cell S-type anion- and Ca2 + permeable channels and stomatal closure. PLoS Bio/ 2006, 4:e327.

92. Munemasa S, Hossain MA, Nakamura Y, Mori IC, Murata Y: The Arabidopsis calcium-dependent protein kinase, CPK6, functions as a positive regulator of methyl jasmonate signaling in guard cells. Plant Physiol 2011, 155:553-561

93. Zhu S-Y, Yu X-C, Wang X-J, Zhao R, Li Y, Fan R-C, Shang Y, Du S-Y, Wang X-F, Wu F-Q, Xu Y-H, Zhang $X-Y$, Zhang D-P: Two calcium-dependent protein kinases, CPK4 and CPK11, regulate abscisic acid signal transduction in Arabidopsis. Plant Cell 2007, 19:3019-3036.

94. Khan M, Rozhon W, Bigeard J, Pflieger D, Husar S, Pitzschke A, Teige M, Jonak C, Hirt H, Poppenberger B: Brassinosteroid-regulated GSK3/shaggylike kinases phosphorylate mitogen-activated protein (MAP) kinase kinases, which control stomata development in Arabidopsis thaliana. J Biol Chem 2013, 288:7519-7527.

95. Kim T-W, Michniewicz M, Bergmann DC, Wang Z-Y: Brassinosteroid regulates stomatal development by GSK3-mediated inhibition of a MAPK pathway. Nature 2012, 482:419-422.

96. Champion A, Picaud A, Henry Y: Reassessing the MAP3K and MAP4K relationships. Trends Plant Sci 2004, 9:123-129.

97. Huang Y, Li H, Hutchison CE, Laskey J, Kieber JJ: Biochemical and functional analysis of CTR1, a protein kinase that negatively regulates ethylene signaling in Arabidopsis. Plant J 2003, 33:221-233.

98. Liu Y, Bassham DC: TOR is a negative regulator of autophagy in Arabidopsis thaliana. PLoS One 2010, 5:e11883.

99. Díaz-Troya S, Pérez-Pérez ME, Florencio FJ, Crespo JL: The role of TOR in autophagy regulation from yeast to plants and mammals. Autophagy 2008, 4:851-865.

100. Rudolf F, Pelet S, Peter M: Regulation of MAPK Signaling in Yeast. In StressActivated Protein Kinases, 20. Edited by Posas F, Nebreda AR. Berlin: Springer; 2008:187-204

101. Zheng CF, Guan KL: Activation of MEK family kinases requires phosphorylation of two conserved Ser/Thr residues. EMBO J 1994, 13:1123-1131.

102. Kim T-W, Guan S, Sun Y, Deng Z, Tang W, Shang J-X, Sun Y, Burlingame AL, Wang Z-Y: Brassinosteroid signal transduction from cell surface receptor kinases to nuclear transcription factors. Nat Cell Biol 2009, 11:1254-1260.

103. Pokhilko A, Fernández AP, Edwards KD, Southern MM, Halliday KJ, Millar AJ: The clock gene circuit in Arabidopsis includes a repressilator with additional feedback loops. Mol Syst Biol 2012, 8:574.

104. Cheong JK, Virshup DM: Casein kinase 1: complexity in the family. Int J Biochem Cell Biol 2011, 43:465-469.

105. Behrend L, Stöter M, Kurth M, Rutter G, Heukeshoven J, Deppert W, Knippschild U: Interaction of casein kinase 1 delta (CK1delta) with post-Golgi structures, microtubules and the spindle apparatus. Eur J Cell Biol 2000, 79:240-251

106. Ben-Nissan G, Cui W, Kim D-J, Yang Y, Yoo B-C, Lee J-Y: Arabidopsis casein kinase 1 -like 6 contains a microtubule-binding domain and affects the organization of cortical microtubules. Plant Physiol 2008, 148:1897-1907.

107. Hirota T, Lee JW, Lewis WG, Zhang EE, Breton G, Liu X, Garcia M, Peters EC, Etchegaray J-P, Traver D, Schultz PG, Kay SA: High-throughput chemical screen identifies a novel potent modulator of cellular circadian rhythms and reveals CKla as a clock regulatory kinase. PLOS Biol 2010, 8:e1000559.

108. Lee J-Y: Versatile casein kinase 1. Plant Signal Behav 2009, 4:652-654

109. Syed S, Saez L, Young MW: Kinetics of doubletime kinase-dependent degradation of the Drosophila period protein. J Biol Chem 2011, jbc.M111.243618.

110. Querfurth C, Diernfellner ACR, Gin E, Malzahn E, Höfer T, Brunner M: Circadian conformational change of the Neurospora clock protein FREQUENCY triggered by clustered hyperphosphorylation of a basic domain. Mol Cell 2011, 43:713-722.

111. Adl SM, Simpson AGB, Lane CE, Lukeš J, Bass D, Bowser SS, Brown MW Burki F, Dunthorn M, Hampl V, Heiss A, Hoppenrath M, Lara E, Le Gall L, Lynn DH, McManus H, Mitchell EAD, Mozley-Stanridge SE, Parfrey LW, Pawlowski J, Rueckert S, Shadwick RS, Shadwick L, Schoch CL, Smirnov A, Spiegel FW: The revised classification of eukaryotes. J Eukaryot Microbiol 2012, 59:429-493.

112. Meggio F, Pinna LA: One-thousand-and-one substrates of protein kinase CK2? FASEB J 2003, 17:349-368 
113. van Ooijen G, Millar AJ: Non-transcriptional oscillators in circadian timekeeping. Trends Biochem Sci 2012, 37:484-492.

114. Lu SX, Liu H, Knowles SM, Li J, Ma L, Tobin EM, Lin C: A role for protein kinase CK2 alpha subunits in the Arabidopsis circadian clock. Plant Physiol 2011.

115. Sugano S, Andronis C, Green RM, Wang ZY, Tobin EM: Protein kinase CK2 interacts with and phosphorylates the Arabidopsis circadian clockassociated 1 protein. Proc Natl Acad Sci U S A 1998, 95:11020-11025.

116. Sugano S, Andronis C, Ong MS, Green RM, Tobin EM: The protein kinase CK2 is involved in regulation of circadian rhythms in Arabidopsis. PNAS 1999, 96:12362-12366.

117. Mehra A, Shi M, Baker CL, Colot HV, Loros JJ, Dunlap JC: A role for casein kinase 2 in the mechanism underlying circadian temperature compensation. Cell 2009, 137:749-760.

118. Portolés S, Más $P$ : The functional interplay between protein kinase CK2 and CCA1 transcriptional activity is essential for clock temperature compensation in Arabidopsis. PLoS Genet 2010, 6:e1001201.

119. Lin J-M, Kilman VL, Keegan K, Paddock B, Emery-Le M, Rosbash M, Allada R: A role for casein kinase $2 a$ in the Drosophila circadian clock. Nature 2002, 420:816-820.

120. Akten B, Jauch E, Genova GK, Kim EY, Edery I, Raabe T, Jackson FR: A role for CK2 in the Drosophila circadian oscillator. Nat Neurosci 2003, 6:251-257.

121. Maier B, Wendt S, Vanselow JT, Wallach T, Reischl S, Oehmke S, Schlosser A, Kramer A: A large-scale functional RNAi screen reveals a role for CK2 in the mammalian circadian clock. Genes Dev 2009, 23:708-718.

122. Lowrey PL, Shimomura K, Antoch MP, Yamazaki S, Zemenides PD, Ralph MR, Menaker M, Takahashi JS: Positional syntenic cloning and functional characterization of the mammalian circadian mutation tau. Science 2000 288:483-491.

123. Gallego M, Eide EJ, Woolf MF, Virshup DM, Forger DB: An opposite role for tau in circadian rhythms revealed by mathematical modeling. Proc Nat Acad Sci U S A 2006, 103:10618-10623.

124. Jones CR, Campbell SS, Zone SE, Cooper F, DeSano A, Murphy PJ, Jones B, Czajkowski L, Ptácek LJ: Familial advanced sleep-phase syndrome: A short-period circadian rhythm variant in humans. Nat Med 1999 5:1062-1065.

125. Toh $\mathrm{KL}$, Jones $\mathrm{CR}$, He Y, Eide EJ, Hinz WA, Virshup DM, Ptácek $L$, Fu YH: An hPer2 phosphorylation site mutation in familial advanced sleep phase syndrome. Science 2001, 291:1040-1043.

126. Xu Y, Padiath QS, Shapiro RE, Jones CR, Wu SC, Saigoh N, Saigoh K, Ptácek LJ, Fu Y-H: Functional consequences of a CKIdelta mutation causing familial advanced sleep phase syndrome. Nature 2005, 434:640-644.

127. Khadaroo B, Robbens S, Ferraz C, Derelle E, Eychenié S, Cooke R, Peaucellier G, Delseny M, Demaille J, van de Peer Y, Picard A, Moreau H: The first green lineage cdc25 dual-specificity phosphatase. Cell Cycle 2004, 3:513-518.

128. Fischer S, Brunk BP, Chen F, Gao X, Harb OS, lodice JB, Shanmugam D, Roos DS, Stoeckert CJ: Using OrthoMCL to Assign Proteins to OrthoMCL-DB Groups or to Cluster Proteomes Into New Ortholog Groups. In Current Protocols in Bioinformatics. Edited by Bateman A, Pearson WR, Stein LD, Stormo GD, Yates JR. Hoboken: John Wiley \& Sons, Inc; 2002.

129. Vilella AJ, Severin J, Ureta-Vidal A, Heng L, Durbin R, Birney E: EnsemblCompara GeneTrees: complete, duplication-aware phylogenetic trees in vertebrates. Genome Res 2009, 19:327-335.

130. Rasko DA, Myers GS, Ravel J: Visualization of comparative genomic analyses by BLAST score ratio. BMC Bioinformatics 2005, 6:2.

131. Eddy SR: Accelerated Profile HMM Searches. PLoS Comput Biol 2011, 7:e1002195.

132. Finn RD, Mistry J, Tate J, Coggill P, Heger A, Pollington JE, Gavin OL, Gunasekaran P, Ceric G, Forslund K, Holm L, Sonnhammer ELL, Eddy SR, Bateman A: The Pfam protein families database. Nucl Acids Res 2010, 38(Suppl 1):D211-D222.

133. Hunter T, Plowman GD: The protein kinases of budding yeast: six score and more. Trends Biochem Sci 1997, 22:18-22.

134. Katoh K, Toh H: Parallelization of the MAFFT multiple sequence alignment program. Bioinformatics 2010, 26:1899-1900.

135. Waterhouse AM, Procter JB, Martin DMA, Clamp M, Barton GJ: Jalview Version 2-a multiple sequence alignment editor and analysis workbench. Bioinformatics 2009, 25:1189-1191.
136. Troshin PV, Procter JB, Barton GJ: Java bioinformatics analysis web services for multiple sequence alignment-JABAWS:MSA. Bioinformatic 2011, 27:2001-2002.

137. Penn O, Privman E, Landan G, Graur D, Pupko T: An alignment confidence score capturing robustness to guide tree uncertainty. Mol Biol Evol 2010, 27:1759-1767

138. Stamatakis $A$, Hoover $P$, Rougemont J: A rapid bootstrap algorithm for the RAxML Web servers. Syst Biol 2008, 57:758-771.

139. Whelan S, Goldman N: A general empirical model of protein evolution derived from multiple protein families using a maximum-likelihood approach. Mol Biol Evol 2001, 18:691-699.

140. Cox J, Mann M: MaxQuant enables high peptide identification rates, individualized p.p.b.-range mass accuracies and proteome-wide protein quantification. Nat Biotechnol 2008, 26:1367-1372.

\section{doi:10.1186/1471-2164-15-640}

Cite this article as: Hindle et al:: The reduced kinome of Ostreococcus tauri: core eukaryotic signalling components in a tractable model species. BMC Genomics 2014 15:640

\section{Submit your next manuscript to BioMed Central and take full advantage of:}

- Convenient online submission

- Thorough peer review

- No space constraints or color figure charges

- Immediate publication on acceptance

- Inclusion in PubMed, CAS, Scopus and Google Scholar

- Research which is freely available for redistribution 